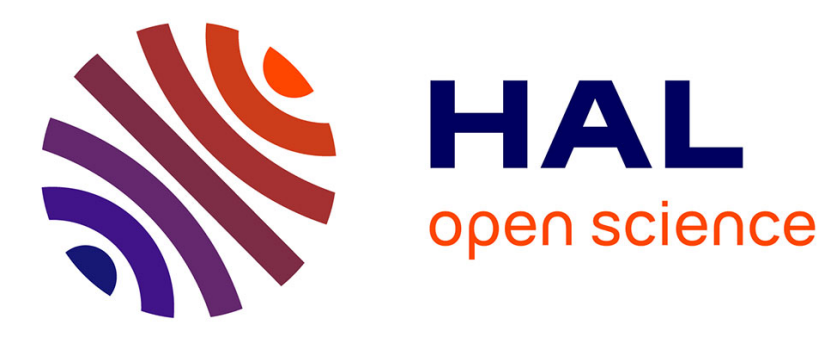

\title{
Effective construction of algebraic geometry codes
}

G. Hache, Dominique Le Brigand

\section{To cite this version:}

G. Hache, Dominique Le Brigand. Effective construction of algebraic geometry codes. [Research Report] RR-2267, INRIA. 1994. inria-00074404

\section{HAL Id: inria-00074404 https://hal.inria.fr/inria-00074404}

Submitted on 24 May 2006

HAL is a multi-disciplinary open access archive for the deposit and dissemination of scientific research documents, whether they are published or not. The documents may come from teaching and research institutions in France or abroad, or from public or private research centers.
L'archive ouverte pluridisciplinaire HAL, est destinée au dépôt et à la diffusion de documents scientifiques de niveau recherche, publiés ou non, émanant des établissements d'enseignement et de recherche français ou étrangers, des laboratoires publics ou privés. 


\section{Effective Construction of Algebraic Geometry Codes}

Gaétan HACHÉ

Dominique Le BRIGAND

$\mathrm{N}^{\circ} 2267$

Mai 1994

PROGRAMME 2

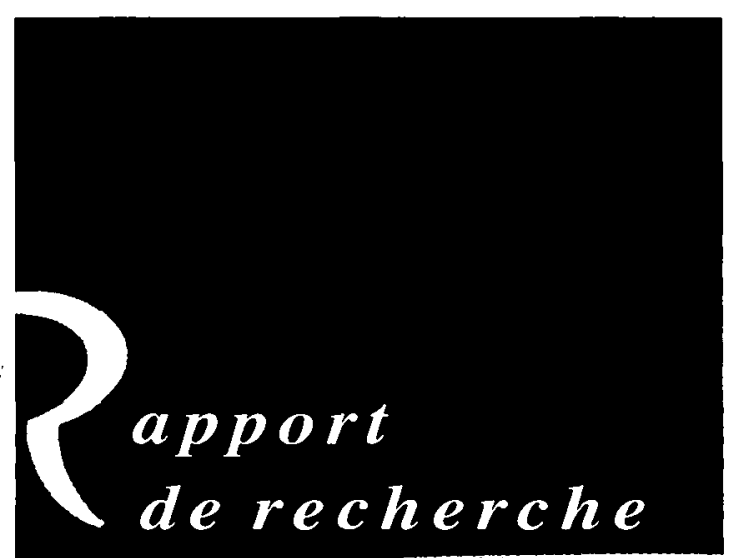




\title{
Effective Construction of Algebraic Geometry Codes
}

\section{Construction Effective des Codes Géométriques}

\author{
Gaétan Haché* and Dominique Le Brigand ${ }^{\dagger}$
}

\begin{abstract}
We intend to show that algebraic geometry codes (AG-codes, introduced by Goppa in 1977 [5]) can be constructed easily using blowing-up theory. This work is based on a paper by Le Brigand and Risler [12]. Given a plane curve, we desingularize the curve by means of blowing-up, and then using the desingularisation trees and the monoidal transformations associated to the blowing-up morphisms, we compute the adjoint divisor of the curve. Finaly we show how to use the algorithm of Brill-Noether to compute a basis of the vector space associated to a divisor of the curve. Two examples of constructions of AG-codes are given at the end.
\end{abstract}

RESUME Nous voulons montrer que les codes géométriques (introduits par Goppa en 1977 [5]) peuvent être construits sans trop de difficulté en utilisant la théorie des éclatements de points. Ce travail est essentiellement basé sur l'article de Le Brigand et Risler [12]. Etant donnée une courbe plane, on procède à une désingularisation de la courbe par éclatements des points singuliers et ensuite, en utilisant les arbres de désingularisations et les transformations monoïdales associées aux morphismes d'éclatements de points, on calcule le diviseur d'adjonction de la courbe. Finalement on montre comment utiliser l'algorithme de BrillNoether pour obtenir une base de l'espace vectoriel associé à un diviseur de la courbe. Deux exemples de constructions de codes géométriques sont donnés à la fin.

*Projet CODES, INRIA-Rocquencourt, Domaine de Voluceau - BP 105, 78153 Le Chesnay Cedex, France. Email: gaetan.hache@inria.fr

tUniversité Pierre et Marie Curie, Paris VI, France. Chercheur extérieur au projet CODES. Email: dominique.lebrigand@inria.fr 


\section{Introduction}

The opinion that algebraic geometry language makes algeb"aic geometry codes (AG-codes) construction very difficult seems to be widely spread in coding theory community. It seems that computing with a plane curve having non ordinary singular points is not well known. We would like to show that it is easy to compute the genus of any singular plane curve, to find a basis of the $k$-vector space $\mathcal{L}(D)$, where $D$ is any divisor on the smooth model of such a curve (even if the support of $D$ contains places above a singular point) and to evaluate functions of the function field at any rational point of the smooth model. We need all this in the construction of an algebraic geometry code. We propose an effective method using blowing-up theory; of course, there are other methods (see $[16,1,13]$ ), but we think that this one is computationally easy even if it may require field extensions. The algorithms have been implemented in Axiom, but we do not want to discuss here their complexity. This paper is mostly based on [12]. It is organized as follows. Section 2 fixes the notations and definitions of algebraic geometry codes. In Section 3, we recall the blowing-up theory, which leads to the desingularization of any plane curve. In Section 4 , we give the construction of a basis for the $k$-vector space $\mathcal{L}(D)$ associated to a divisor $D$. Section 5 precises some algorithms we need for the construction of AG-codes. Finally, in Section 6, we give examples of codes using two different curves.

\section{Preliminaries - Notations}

One can find basic facts concerning algebraic curves in [15] or [14, (Appendix B)] where the function fields point of view is treated. We will use generally the same notations as [14]. Let $k$ be the finite field $\mathbb{F}_{q}, \bar{k}$ its algebraic closure, $C$ an affine curve defined and absolutely irreducible over $k, P$ a rational point of $C$. We can suppose without loss of generality that $P=(0,0)$ in the affine coordinates $(x, y)$ and $f(x, y)=0$ is the equation of $C$. The affine coordinate ring of $C$ is the integral domain

$$
\Gamma(C)=k[x, y] /\langle f\rangle .
$$

Its function field $k(C)$ is the quotient field of $\Gamma(C)$, that is

$$
k(C)=\{g / h \mid g, h \in \Gamma(C), h \neq 0\} .
$$

Now, let $\mathcal{X}$ be a smooth projective curve defined and absolutely irreducible over $k$. If $\mathcal{C}$ is a projective plane model of $\mathcal{X}$ with equation $F=0$, where $F \in k[X, Y, Z]$ is an absolutely irreducible form, then its homogeneous coordinate ring is

$$
\Gamma(\mathcal{C})=k[X, Y, Z] /\langle F\rangle
$$

For $G \in k[X, Y, Z]$ a form, we denote by $\bar{G}$ its residual image in $\Gamma(\mathcal{C})$. The function field $K / k$ of $\mathcal{X}$ is $k$-isomorphic to $k(\mathcal{C}) / k$ where

$$
k(\mathcal{C})=\{\bar{G} / \bar{H} \mid G, H \in k[X, Y, Z], \text { forms of equal degree, } \bar{H} \neq 0\} .
$$


We identify $K / k$ with $k(\mathcal{C}) / k$. Suppose that $P=(0: 0: 1)$ in homogeneous coordinates $(X: Y: Z)$ and set $f(x, y)=F(x, y, 1)=0$. Then $f=0$ is the equation of the affine part $C_{P}$ of $\mathcal{C}$ at $P$ and $k(\mathcal{C})$ is naturally isomorphic to $k\left(C_{P}\right)$. The local ring of $\mathcal{C}$ at $P$ is

$$
\mathcal{O}_{P}(\mathcal{C})=\left\{g / h \mid g, h \in \Gamma\left(C_{P}\right), h(P) \neq 0\right\}
$$

and its unique maximal ideal is

$$
\mathcal{M}_{P}(\mathcal{C})=\left\{g / h \in \mathcal{O}_{P}(\mathcal{C}) \mid g(P)=0\right\}
$$

$\mathcal{O}_{P}(\mathcal{C})$ is a discrete valuation ring if and only if $P$ is a simple point of $\mathcal{C}$. Let $Q$ be any $k$-rational point of $k(\mathcal{C})$; assume for instance that $Q$ is in the affine open set defined by $Z \neq 0$ and let $G \in k[X, Y, Z]$ be a form of degree $d$. We associate to $G$ the element of $\mathcal{O}_{Q}(\mathcal{C})$

$$
\bar{G}^{Q}=\bar{G} / \bar{Z}^{d} .
$$

More generally, a place $\wp$ of $K / k$ is the maximal ideal of some discrete valuation ring $\mathcal{O}_{\wp}$

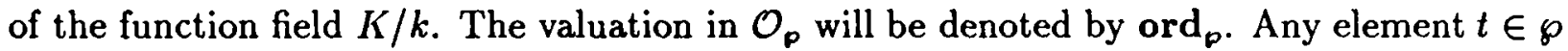
such that $\wp=\langle t\rangle$ is called a local parameter at $\wp$ and $\operatorname{ord}_{p}(t)=1$. If $\wp$ is a place of degree one, we will say that $\wp$ is a $k$-rational point of $\mathcal{X}$. In particular, to any simple $k$-rational point $P$ of the plane model $\mathcal{C}$, corresponds a unique place $\wp$ of degree one of $K / k$ and we will identify $P$ with $\wp$. If $P$ is a $k$-rational singular point of $\mathcal{C}$, then there is a finite number of places $\wp$ of $K / k$ such that

$$
\mathcal{O}_{P}(\mathcal{C}) \subset \mathcal{O}_{p}
$$

we say that $\wp$ is above $P$ and write $\wp \succ P$. Note that a place $\wp$ above a $k$-rational singular point is not necessarily of degree one. If $\operatorname{deg} \wp=r$, denoting by $k_{r}$ the extension of $k$ of degree $r$, we have $\varphi=Q_{1}+\cdots+Q_{r}$ where each $Q_{i}$ is a place ${ }^{1}$ of $\bar{k}(\mathcal{C}) / \bar{k}$ and $Q_{1}, \ldots, Q_{r}$ are conjugated over $k$ by $\mathcal{G} a l(\bar{k} / k)$. In fact the places $Q_{i}, i=1, \ldots, r$, can be considered as places of degree one of $k_{r}(\mathcal{C}) / k_{r}$. If $u \in k(\mathcal{C})$, we denote also by $u$ its image in $k_{r}(\mathcal{C})$ by the canonical embedding of $k(\mathcal{C})$ in $k_{r}(\mathcal{C})$ and we have

$$
\operatorname{ord}_{p}(u)=\operatorname{ord}_{Q_{i}}(u), \forall i=1, \ldots, r .
$$

Conversely, if $Q$ is any place of $\bar{k}(\mathcal{C}) / \bar{k}$, it belongs to a unique place $\wp$ of $K$; suppose again that $\operatorname{deg} \varphi=r$ and let $\sigma$ be a generator of $\mathcal{G} a l\left(k_{r} / k\right)$. Then $i_{Q}=r$ is the smallest positive integer such that $Q^{\sigma^{\circ} Q}=Q$ and we have

$$
\wp=\sum_{j=0}^{i_{Q}-1} Q^{\sigma^{j}} .
$$

Let $k^{\prime}$ be any extension of $k$ (or $k$ itself) and let $\wp$ be a place of $k^{\prime}(\mathcal{C}) / k^{\prime}$; for brevity, we shall refer to $\wp$ as a place of $k^{\prime}(\mathcal{C})$. Likewise, if $D$ is a divisor of $k^{\prime}(\mathcal{C}) / k^{\prime}$, we shall say that $D$ is a $k^{\prime}$-rational divisor of $k^{\prime}(\mathcal{C})$.

Finally, let us recall the definitions of algebraic geometry codes (cf. [15, p.266-]).

\footnotetext{
${ }^{1}$ Note that all the places of $\bar{k}(\mathcal{C}) / \bar{k}$ are of degree one.
} 
Definition 2.1 Let $\mathcal{X}$ be a smooth projective curve defined and absolutely irreducible over the finite field $k=\mathbb{F}_{q}$. We denote by $K / k$ its function field and let $\mathcal{P}$ and $D$ be divisors of $K / k$ with disjoint supports and

$$
\mathcal{P}=P_{1}+\cdots+P_{n}
$$

where $P_{i}, i=1, \ldots, n$, are distinct $k$-rational points of $\mathcal{X}$. The algebraic geometry code $C_{L}(\mathcal{X}, \mathcal{P}, D)$ is the image of the $k$-linear map

$$
e v_{\mathcal{P}}: \mathcal{L}(D) \longrightarrow k^{n}
$$

such that

$$
e v_{\mathcal{P}}(u)=\left(u\left(P_{1}\right), \ldots, u\left(P_{n}\right)\right) .
$$

The code $C_{L}(\mathcal{X}, \mathcal{P}, D)$ has lenght $n$, dimension $\ell(D)-\ell(D-\mathcal{P})(\ell(D)$ if $\operatorname{deg} D<n)$ and minimum distance $d$ such that $d \geq n-\operatorname{deg} D ; d^{*}=n-\operatorname{deg} D$ is the designed distance of the code $C_{L}$. A generator matrix for $C_{L}(\mathcal{X}, \mathcal{P}, D)$ can be obtained knowing a basis for $\mathcal{L}(D)$ and a procedure for the evaluation of functions at the rational points $P_{i}$.

There is another construction of algebraic geometry codes

Definition 2.2 Let $\mathcal{X}$ be a smooth projective curve defined and absolutely irreducible over the finite field $k=\mathbb{F}_{q}$. We denote by $K / k$ its function field and let $\mathcal{P}$ and $D$ be divisors of $K / k$ with disjoint supports and

$$
\mathcal{P}=P_{1}+\cdots+P_{n}
$$

where $P_{i}, i=1, \ldots, n$, are distinct $k$-rational points of $\mathcal{X}$. The algebraic geometry code $C_{\Omega}(\mathcal{X}, \mathcal{P}, D)$ is the image of the $k$-linear map

$$
\operatorname{resp}: \Omega(\mathcal{P}-D) \longrightarrow k^{n}
$$

such that

$$
\operatorname{resp}_{p}(\omega)=\left(\operatorname{res}_{P_{1}}(\omega), \ldots, \operatorname{res}_{p_{n}}(\omega)\right) .
$$

The codes $C_{L}(\mathcal{X}, \mathcal{P}, D)$ and $C_{n}(\mathcal{X}, \mathcal{P}, D)$ are dual to each other; so a generator matrix for one of them gives a parity check matrix for the other. Finally, we recall that construction of bases for $k$-vector spaces $\mathcal{L}(G)$, where $G$ is any divisor, is essential for presently existing decoding algorithms of algebraic geometry codes.

\section{Blowing-up}

The blowing-up theory is developed in many books, see for instance $[7,15]$. The universal domain $k$ is usually supposed to be an algebraically closed field. In $[6,8] k$ is an arbitrary field. The blowing-up process gives key informations concerning the smooth model of a given singular plane curve and its function field. 


\subsection{Definitions}

Definition 3.1 ( $[7, p .28])$ Blowing-up a point in the affine space. Let $k$ be any field and let $P$ be the point $P=(0,0)$ in $\mathbb{A}^{2}(k)$. We consider the sub-variety $W_{1}$ of $\mathbb{A}^{2} \times \mathbb{P}^{1}$ defined by

$$
W_{1}=\left\{(x, y) \times\left(x_{1}: y_{1}\right) \in \mathbb{A}^{2} \times \mathbb{P}^{1} \mid x y_{1}-y x_{1}=0\right\}
$$

where $\left(x_{1}: y_{1}\right)$ are the homogeneous coordinates in $\mathbb{P}^{1}$. Then the blowing-up $\pi$ of $P$ in $\mathbb{A}^{2}$ is the restriction to $W_{1}$ of the canonical projection from $\mathbb{A}^{2} \times \mathbb{P}^{1}$ to $\mathbb{A}^{2}$.

Properties of $\pi$.

1. $W_{1}$ is a smooth, irreducible surface defined over $k$.

2. If $Q$ is any point in $\mathbb{A}^{2}(\bar{k})$ distinct from $P$, then $\pi^{-1}(Q)$ reduces to a single point; more precisely if $Q=(a, b) \neq(0,0)$ then

(a) $\pi^{-1}(Q)=\{(a, b) \times(1: b / a)\}$, if $a \neq 0$;

(b) $\pi^{-1}(Q)=\{(a, b) \times(a / b: 1)\}$, if $b \neq 0$.

(c) If $Q$ is $k$-rational $\left(Q \in \mathbb{A}^{2}(k)\right)$, then so is $\pi^{-1}(Q)$. More generally, there exists an isomorphism

$$
W_{1} \backslash \pi^{-1}(P) \longrightarrow \mathbb{A}^{2} \backslash\{P\}
$$

defined over $k$.

3. $\pi^{-1}(P)$ is isomorphic to $\mathbb{P}^{1}$; the points of $\pi^{-1}(P)$ are in one-to-one correspondence with the lines in $\mathbb{A}^{2}(\bar{k})$ passing by $P$.

One may generalize the definition of blowing-up as follows. Let $S$ be a smooth projective surface defined over $k$ and having the property that each point $Q$ of $S$ has an open neighbourhood $U_{Q}$ isomorphic to $\mathbb{A}^{2}$. Let $P$ be a $k$-rational point of $S$. Then there exists a unique smooth projective surface $S_{1}$ defined over $k$ with the same property as $S$ and there is a morphism

$$
\pi: S_{1} \rightarrow S
$$

such that $S_{1} \backslash \pi^{-1}(P)$ is isomorphic to $S \backslash\{P\}$ and $\pi^{-1}(P)$ is isomorphic to $\mathbb{P}^{1}$. If $P=(0,0)$ in the local coordinates of $U_{P}$, then the restriction of $\pi$ above $U_{P}$ coincides with the morphism considered in the preceding definition. The morphism $\pi$ is the blowing-up of $P$ in $S$.

\subsection{Desingularization of a plane projective curve}

Let $S_{0}=\mathbb{P}^{2}(k)$, let $\mathcal{C}$ be a projective plane curve defined and absolutely irreducible over $k$ and let $P$ be a $k$-rational singular point of $\mathcal{C}$. 
Definitions 3.1 Let $\pi$ be the blowing-up morphism of $P$ in $S_{0}$.

- The total transform of $\mathcal{C}$ by $\pi$ is the inverse image of $\mathcal{C}$ by $\pi$.

- The strict transform $\mathcal{C}_{1}$ of $\mathcal{C}$ by $\pi$ is the closure of $\pi^{-1}(\mathcal{C} \backslash\{P\})$ in $S_{1} ; \mathcal{C}_{1}=$ $\overline{\pi^{-1}(\mathcal{C} \backslash\{P\})}$.

- The exceptional line is $\mathcal{E}_{1}=\pi^{-1}(P)$.

- The infinitely near points of $P$ by $\pi$ are the common points of $\mathcal{E}_{1}$ and $\mathcal{C}_{1}$.

Using the properties of $\pi$, we have that $\mathcal{C}_{1} \backslash \pi^{-1}(P)$ is isomorphic to $\mathcal{C} \backslash\{P\}$. The curves $\mathcal{C}$ and $\mathcal{C}_{1}$ are birationally equivalent and $\pi$ induces a $k$-isomorphism from $k(\mathcal{C})$ to $k\left(\mathcal{C}_{1}\right)$. The point $P$ is blown up into a projective line $\mathcal{E}_{1}$. We will show later that the number of infinitely near points equals the number of distinct tangents to $\mathcal{C}$ at $P$. Notice that these points may belong to a finite extension of $k$. The singular points of $\mathcal{C}_{1}$ are

1. the singular points of $\mathcal{C} \backslash\{P\}$, if any, using the identification of $\mathcal{C}_{1} \backslash \pi^{-1}(P)$ with $\mathcal{C} \backslash\{P\}$,

2. eventually, the infinitely near points of $P$ by $\pi$.

If $Q$ is a singular point of $\mathcal{C}_{1}$ we blow up $Q$. We may have to consider a finite extension $k^{\prime}$ of $k$ if $Q$ is not $k$-rational. After a finite number of blowing-ups we will obtain a smooth curve. We have the following result:

Theorem 3.1 Let $\mathcal{C}_{0}$ be a singular projective plane curve defined and absolutely irreducible over $k$; we set $S_{0}=\mathbb{P}^{2}(k)$. Then, eventually over a finite algebraic extension $k^{\prime}$ of $k$, there exist smooth projective surfaces $S_{i}(i=1, \ldots, n)$ defined over $k^{\prime}$ and a finite sequence of morphisms

$$
S_{n} \stackrel{\pi_{n}}{\rightarrow} S_{n-1} \stackrel{\pi_{n-1}}{\rightarrow} \cdots \stackrel{\pi_{3}}{\rightarrow} S_{1} \stackrel{\pi_{1}}{\rightarrow} S_{0}
$$

where, for $i=1, \ldots, n, \pi_{i}$ is the blowing-up of a $k^{\prime}$-rational point $P_{i-1}$ of $S_{i-1}$ and if we denote by $\mathcal{C}_{i}$ the strict transform $\mathcal{C}_{i-1}, P_{i-1}$ is a singular point of $\mathcal{C}_{i-1}$. The last strict transform $\mathcal{C}_{n}$ is a smooth curve defined over $k^{\prime}$ which is birationally equivalent to $\mathcal{C}_{0}$; it is the smooth model $\mathcal{X}$ of $\mathcal{C}$ and we say that $\mathcal{C}$ has a desingularization over $k^{\prime}$.

Let us see now precisely what we have to do in order to blow up a point $P$ of a plane curve ( $P$ singular or not).

1. Local equation of the curve at $P$. Let $\mathcal{C}:\{F=0\}$ be a projective plane curve defined over $k$ and let $P$ be a $k$-rational point of $\mathcal{C}$. Up to a linear change of projective coordinates $(X: Y: Z)$, we can have $P=(0: 0: 1)$; so $x=X / Z$ et $y=Y / Z$ are local coordinates at $P$ in the affine open set defined by $Z \neq 0$ and the affine equation of $\mathcal{C}$ at $P$ is

$$
f(x, y)=F(x, y, 1)=0
$$

We can write

$$
f(x, y)=f_{r}(x, y)+f_{r+1}(x, y)+\cdots+f_{n}(x, y)
$$


where the $f_{i}$ 's are homogeneous forms of degree $i ; f_{r}$ is the initial form of $f, r=$ $\mathbf{m}_{P}(\mathcal{C})=\mathbf{m}_{P}(F) \geq 1$ is the multiplicity of the point $P . P$ is singular (resp. simple) if and only if $r>1$ (resp. $r=1$ ). The factorization of $f_{r}$ in $\bar{k}[x, y]$ is

$$
f_{r}(x, y)=\prod_{i=1}^{s} L_{i}(x, y)^{n_{i}}
$$

with $L_{i}(x, y)=a_{i} x+b_{i} y$ and $\sum_{i=1}^{s} n_{i}=r$. The curve $\mathcal{C}$ has $s$ distinct tangents at $P$ whose equations are $L_{i}=0, i=1, \ldots, s$.

2. Strict transform of the curve by the blowing-up of $P$. The equation in $W_{1}$ of the total transform of $\mathcal{C}$ by $\pi$ is

$$
\left\{\begin{aligned}
f(x, y) & =0 \\
x y_{1}-y x_{1} & =0 .
\end{aligned}\right.
$$

$\mathbb{P}^{1}$ is covered by two open sets: one is defined by $x_{1} \neq 0$, the other by $y_{1} \neq 0$. So $W_{1}$ is covered by two open sets isomorphic to $\mathbb{A}^{2}$

(a) the first one, denoted by $U_{x}$, corresponds to $x_{1}=1$. Identifying $U_{x}$ with $\mathbb{A}^{2}$, we have that $\left(x, y_{1}\right)$ are local coordinates in $U_{x}$ and the restriction $\pi_{x}$ of $\pi$ to $U_{x}$ is given by

$$
\pi_{x}\left(x, y_{1}\right)=\left(x, x y_{1}\right) ;
$$

(b) the second one, denoted by $U_{y}$, corresponds to $y_{1}=1$. Identifying $U_{y}$ with $\mathbb{A}^{2}$, we have that $\left(x_{1}, y\right)$ are local coordinates in $U_{y}$ and the restriction $\pi_{y}$ of $\pi$ to $U_{y}$ is given by

$$
\pi_{y}\left(x_{1}, y\right)=\left(x_{1} y, y\right) .
$$

The equation in $U_{x}$ of the total transform of $\mathcal{C}$ is

$$
0=f\left(x, x y_{1}\right)=x^{r} f_{x}\left(x, y_{1}\right)
$$

where $f_{x}$ is a polynomial in $k\left[x, y_{1}\right]$ such that $f_{x}\left(0, y_{1}\right) \neq 0$. We obtain two curves. One of them corresponds to $x=0$; this is the local equation in $U_{x}$ of the exceptional line $\mathcal{E}_{1}$. The other is defined by $f_{x}\left(x, y_{1}\right)=0$; this is the local equation in $U_{x}$ of the strict transform $\mathcal{C}_{1}$. The coordinates of the common points of $\mathcal{C}_{1}$ and $\mathcal{E}_{1}$ in $U_{x}$ are $(0, \alpha)$, where $\alpha$ is a root of

$$
f_{x}(0, T)=\prod_{i=1}^{\infty}\left(a_{i}+b_{i} T\right)^{n_{i}}=0,
$$

so $\alpha$ is the slope of a tangent to the curve at $P$. The number of these points is equal to the number of tangents which are distinct from $x=0$.

The equation in $U_{y}$ of the total transform is

$$
0=f\left(y x_{1}, y\right)=y^{r} f_{y}\left(x_{1}, y\right)
$$

where $f_{y}$ is a polynomial in $k\left[x_{1}, y\right]$ such that $f_{y}\left(x_{1}, 0\right) \neq 0$. We obtain two curves defined respectively by $y=0$, the local equation of the exceptional line, and $f_{y}\left(x_{1}, y\right)=$ 
0 , the local equation of the strict transform. The coordinates of the common points of $\mathcal{C}_{1}$ and $\mathcal{E}_{1}$ in $U_{y}$ are $(\beta, 0)$, where $\beta$ is a root of

$$
f_{y}(T, 0)=\prod_{i=1}^{s}\left(a_{i} T+b_{i}\right)^{n_{i}}=0 .
$$

The number of these points equals the number of tangents which are distinct from $y=0$. We eventually recover the points corresponding to the tangents such that $b_{i} \neq 0$ and a new point $Q_{0}=(0,0)$ if $\{x=0\}$ is a tangent. If $\{x=0\}$ is not tangent to the curve at $P$, we obtain all the infinitely near points of $P$ in the open set $U_{x}$ defined by $x_{1}=1$. Otherwise the infinitely near points of $P$ are

(a) all the points $Q=(0, \alpha)$ in $U_{x}$ such that $f_{x}(0, \alpha)=0$;

(b) the point $Q=(0,0)$ in $U_{y}$ if $f_{y}(0,0)=0$.

Notice that if $P$ is a simple point of $\mathcal{C}$, then there is a unique infinitely near point $Q$ which is a simple point of $\mathcal{C}_{1}$. If $P$ is $k$-rational, $Q$ is also $k$-rational. Let $Q$ be any point of $\mathcal{C}_{1}$ and let $(u, v)$ be local coordinates in an affine open neighbourhood $V$ of $Q$ such that $Q=(0,0)$ and $g(u, v)=0$ is the affine equation of $\mathcal{C}_{1}$. We can blow up the point $Q$ the same way as before :

(a) if $Q$ is an infinitely near point of $P$ and $V=U_{x}$, then $(u, v)=\left(x, y_{1}-\alpha\right)$, $g=f_{x}\left(x, y_{1}-\alpha\right) ;$ if $V=U_{y}$, we have $(u, v)=\left(x_{1}, y\right)$ and $g=f_{y}\left(x_{1}, y\right) ;$

(b) if $Q$ corresponds to a point of $\mathcal{C}$ distinct from $P$ by the isomorphism $\mathcal{C}_{1} \backslash \pi^{-1}(P) \rightarrow$ $\mathcal{C} \backslash\{P\}$, we blow up this point considered as a point of $\mathcal{C}$.

3. Desingularization tree (see $[15$, p.230]). Let $\mathcal{C}$ be a plane singular projective curve. We define the desingularization tree $\mathcal{T}$ of $\mathcal{C}$. Each singular point $P$ of the curve is the root of a sub-tree $\mathcal{T}_{P}$ of $\mathcal{T}$ and $P$ is characterized by its coordinates and the local equation of the curve at $P$. To each infinitely near point $Q$ of $P$ corresponds a branch; $Q$ is a node of the sub-tree. To $Q$ is attached informations concerning the open set $U_{x}$ or $U_{y}$ which contains it, the local coordinates at $Q$, functions of those at $P$, and the affine equation of the strict transform. The sub-tree $\mathcal{T}_{P}$ is constructed ${ }^{2}$ recursively: if a node is a singular point of some curve $\mathcal{C}_{i}$, then it is the root of a sub-tree. A branch stops when we obtain a simple point, called a leaf, on the corresponding strict transform.

In our implementation we make the computations in a field $k$ big enough so that all the nodes of the tree are $k$-rational.

\subsection{Monoidal transformation}

Let $P$ be any $k$-rational point of the curve $\mathcal{C}$ and let $f(x, y)=0$ be the equation of $\mathcal{C}$ in an open affine neighbourhood of $P$ such that $P=(0,0)(P$ is not necessarily a singular point of $\mathcal{C}$ ). Let $\mathcal{C}_{1}$ be the strict transform of the curve by the blowing-up of $P$. We have seen

\footnotetext{
${ }^{2}$ In Section 5.2 an algorithm is given.
} 
that the function field $k(\mathcal{C})$ can be identified with the quotient field of $k[x, y] /\langle f\rangle$ and if $f_{x}$ (resp. $f_{y}$ ) is the affine equation of $\mathcal{C}_{1}$ in $U_{x}$ (resp. $U_{y}$ ), we can identify the function field $k\left(\mathcal{C}_{1}\right)$ with the quotient field of $k\left[x, y_{1}\right] /\left\langle f_{x}\right\rangle$ (resp. the quotient field of $k\left[x_{1}, y\right] /\left\langle f_{y}\right\rangle$ ). Using these identifications, we obtain the following $k$-isomorphisms

$$
\begin{array}{llcc}
\pi_{x}^{*}: & k(\mathcal{C}) & \longrightarrow & k\left(\mathcal{C}_{1}\right) \\
g(x, y) / h(x, y) & \mapsto & g\left(x, x y_{1}\right) / h\left(x, x y_{1}\right)
\end{array}
$$

and

$$
\begin{array}{llcc}
\pi_{y}^{*}: & k(\mathcal{C}) & \longrightarrow & k\left(\mathcal{C}_{1}\right) \\
& g(x, y) / h(x, y) & \mapsto & g\left(y x_{1}, y\right) / h\left(y x_{1}, y\right) .
\end{array}
$$

We call $\pi_{x}^{*}$ (resp. $\pi_{y}^{*}$ ) the monoidal transformation of the blowing-up of $P$ with respect to $U_{x}$ (resp. $U_{y}$ ). If $Q$ is an infinitely near point of $P$, then

$$
\pi_{x}^{*}\left(\mathcal{O}_{P}(\mathcal{C})\right) \subset \mathcal{O}_{Q}\left(\mathcal{C}_{1}\right)
$$

and we have a similar result for $\pi_{y}^{*}$. Suppose now that $\mathcal{C}$ has a desingularization over $k^{\prime}, P$ is a singular point and $Q$ is a leaf of $\mathcal{T}_{P}$; then $Q$ is a simple point of some strict transform, denoted by $\mathcal{C}_{Q}$, which can be considered as an affine curve when we restrict ourself to the open neighbourhood isomorphic to $\mathbb{A}^{2}$ containing $Q$. If $\pi_{1}, \pi_{2}, \ldots, \pi_{8}$ is the finite sequence of blowing-ups which leads from $P$ to $Q$, we consider the $k^{\prime}$-isomorphism

$$
\pi_{Q}^{*}: k^{\prime}(\mathcal{C}) \longrightarrow k^{\prime}\left(\mathcal{C}_{Q}\right)
$$

obtained by the composition of the monoidal transformations associated to $\pi_{i}, i=1, \ldots, s$, with respect to the appropriate affine neighbourhoods. Then the leaves $Q$ of the sub-tree $\mathcal{T}_{P}$ are in one-to-one correspondence with the places of $k^{\prime}(\mathcal{C})$ lying over $P$ and

$$
\pi_{Q}^{*}\left(\mathcal{O}_{P}(\mathcal{C})\right) \subset \mathcal{O}_{Q}\left(\mathcal{C}_{Q}\right)
$$

Hence for any place $\wp$ above $P$ there is a unique leaf $Q$ such that

$$
\pi_{Q}^{*}\left(\mathcal{O}_{p}\right)=\mathcal{O}_{Q}\left(\mathcal{C}_{Q}\right)
$$

for any $u \in k^{\prime}(\mathcal{C})$ we have

$$
\operatorname{ord}_{\bullet}(u)=\operatorname{ord}_{Q}\left(\pi_{Q}^{*}(u)\right)
$$

and if $u \in \mathcal{O}_{\mathfrak{p}}$ then

$$
u(\wp)=\pi_{Q}^{*}(u)(Q)
$$

\subsection{The $\wp$-morphism}

Let $\wp$ be any place of $\bar{k}(\mathcal{C})$ and $k^{\prime}$ a finite extension of $k$ such that $\wp$ is place of degree one of $k^{\prime}(\mathcal{C})$. If $\wp=P$ is a simple point of $\mathcal{C}$ we may define, by choosing an affine neighbourhood and up to a linear change of affine coordinates, an affine plane curve $C^{\bullet}$ defined over $k^{\prime}$ and a birational morphism

$$
\phi_{p}: C^{p} \longrightarrow \mathcal{C}
$$


such that

$$
\phi_{\wp}((0,0))=P \text {. }
$$

If $\varphi$ is above a singular point $P$ of $\mathcal{C}$, then by blowing-ups and linear affine changes of coordinates we may also define an affine plane curve $C^{\bullet}$ defined over $k^{\prime}$ and a birational morphism

$$
\phi_{p}: C^{\mathfrak{D}} \longrightarrow \mathcal{C}
$$

such that

$$
\phi_{\triangleright}((0,0))=P \text {. }
$$

In either case, we have a $k^{\prime}$-isomorphism

$$
\phi_{\wp}^{*}: k^{\prime}(\mathcal{C}) \longrightarrow k^{\prime}\left(C^{p}\right)
$$

such that

$$
\phi_{p}^{*}\left(\mathcal{O}_{p}\right)=\mathcal{O}_{(0,0)}\left(C^{p}\right)
$$

We also have another useful property: since linear changes of affine coordinates and blowingups consist in linear and quadratic substitutions, we have for any form $G \in k^{\prime}[X, Y, Z]$

$$
\phi_{\mathfrak{b}}^{*}\left(\bar{G}^{P}\right) \in \Gamma\left(C^{\mathfrak{p}}\right) .
$$

Hence, for the computation of the order or the evaluation of functions at places $\wp$ of $\bar{k}(\mathcal{C})$, it is sufficient to have algorithms defined at the simple point $(0,0)$ of the affine plane curve $C^{\bullet}$. We call the morphism $\phi_{\mathfrak{p}}$ the $\wp$-morphism.

\subsection{Genus of a plane curve}

Let $\mathcal{T}$ be the desingularization tree of the plane curve $\mathcal{C}:\{F=0\}$ and let $m$ be the degree of $F$. Suppose that the tree consists of successive blowing-ups $\pi_{i}, i=1, \ldots, n$, of $n$ points $P_{1}, \ldots, P_{n}$, where, for $i=1, \ldots, n, P_{i}$ is a singular point of $\mathcal{C}_{i}$ of multiplicity $r_{i}$. We have the classical formula for the genus of the curve (see [15, p.229])

$$
g=\frac{(m-1)(m-2)}{2}-\sum_{i=1}^{n} \frac{r_{i}\left(r_{i}-1\right)}{2}
$$

The preceding sum is taken over all the nodes of $\mathcal{T}$ including the singular points of the plane curve.

\subsection{Additional remarks}

Let $k$ be the finite field $\mathbb{F}_{q}$ and $\mathcal{C}$ a projective plane curve defined and absolutely irreducible over $k$. We suppose that the singular points of $\mathcal{C}$ are rational over $k$; if not we replace $k$ by a finite extension. Then the blowing-up of one of them, $P$, is defined on $k$ and birational. The initial form $f_{r}$ of the affine equation of $\mathcal{C}$ at $P$ factors over $k[x, y]$. Let the factorization be

$$
f_{r}=\prod_{j=1}^{t} p_{j}(x, y)^{m_{j}},
$$


where $p_{j}$ is an irreducible polynomial over $k$. We set $r_{j}=\operatorname{deg} p_{j}$ and denote by $k_{j}$ the extension of $k$ of degree $r_{j}$. Using similar notations as in (2) we have

$$
f_{r}(x, y)=\prod_{j=1}^{t} \prod_{i=1}^{r_{j}} L_{i, j}(x, y)^{m_{j}} .
$$

For any $j, j=1, \ldots, t$, the slopes of the tangents $\left\{L_{i, j}=0\right\}, i=1, \ldots, r_{j}$ are conjugated over $k$ and so are the corresponding infinitely near points $Q_{i, j}$. For a fixed $j$, we set $r=r_{j}$, $Q_{i}=Q_{i, j} i=1, \ldots, r$. Since the equation of the strict transform $\mathcal{C}_{1}$ is defined over $k$, if one of the $Q_{i}$ is simple, so are the others; in that case, $\wp=\sum_{i=1}^{r} Q_{i}$ is a place of degree $r$ of $k\left(\mathcal{C}_{1}\right)$. If one of the $Q_{i}$ is singular, so are the others, of equal multiplicity. Further, the sub-trees corresponding to each $Q_{i}$ are, in a sense, conjugated: this means that if $\sigma$ is an element of $\mathcal{G a l}\left(k_{r} / k\right)$ and $Q_{i}^{\sigma}=Q_{l}$, there is a canonical bijection between the sub-trees $T_{Q_{i}}$ and $\mathcal{T}_{Q_{1}}$ which sends each node $N$ of $\mathcal{T}_{Q_{i}}$ to $N^{\sigma}$ which is a node of $\mathcal{T}_{Q_{1}}$. The local equation of the strict transform at $N$ and $N^{\sigma}$ are also conjugated by $\sigma$ and $N$ and $N^{\sigma}$ have equal multiplicity on their respective strict transform. In fact, this is already true for the singular points of $\mathcal{C}$. If $P$ is a singular point which is not $k$-rational and $k_{r}$ is the least extension of $k$ containing the coordinates of $P$, then, since the curve is defined over $k$, the conjugate points of $P$ are singular points of the curve with the same multiplicity as $P$ and their sub-trees are conjugated. 'For this reason we can say that, if the curve is defined over $k$, then its desingularization tree is globally rational over $k$.

In [7, p.163], the definition of a more general blowing-up is given. Theorically it allows to blow up at the same time a singular point and all its conjugates over $k$ and so to stay in the base field $k$. But, at that moment, we cannot find an effective implementation of this theory.

\section{Adjoint curve}

The adjoint curves of an absolutely irreducible projective plane curve $\mathcal{C}$ play a crucial role in the determination of bases for vector spaces $\mathcal{L}(D)$, where $D$ is any divisor of $k(\mathcal{C})$ (see $[6,12])$. An adjoint curve of a plane curve $\mathcal{C}$ is a plane curve passing by the singular points of $\mathcal{C}$ with a "large enough" multiplicity.

\subsection{Adjunction divisor}

In that Section we refer to [6] or [12] for the proofs. Let $\mathcal{C}$ be a curve defined over $k$ which has a desingularization over a finite extension $k^{\prime}$ of $k$ and let $\mathcal{T}$ be its desingularization tree. Suppose that this tree consists of successive blowing-ups $\pi_{i}, i=1, \ldots, n$, of $n k^{\prime}$-rational points $P_{1}, \ldots, P_{n}$, where, for $i=1, \ldots, n, P_{i}$ is a singular point of $\mathcal{C}_{i}\left(\mathcal{C}_{1}=\mathcal{C}\right)$ of multiplicity $r_{i}$. Let $E_{i}$ be the divisor of $k(\mathcal{C})$ associated to the exceptional line $\mathcal{E}_{i}$ of $\pi_{i}$ : we call $E_{i}$ the exceptional divisor of $\pi_{i}$. $\mathcal{E}_{i}$ cuts the strict transform $\mathcal{C}_{i}$ at the infinitely near points of $P_{i}$ and so the support of $E_{i}$ is equal to the set of leaves of the tree $\mathcal{T}_{P_{i}}$. It can be shown, see Lemma 5.1, that its degree is equal to $r_{i}$. Using the preceding notations, we have 
Definitions 4.1 Let $\mathcal{C}:\{F=0\}$ be a projective plane curve defined and absolutely irreducible over a field $k$ and let $\mathcal{T}$ be its desingularization tree defined over a finite extension $k^{\prime}$ of $k$.

- The divisor of $k^{\prime}(\mathcal{C})$ defined by

$$
\mathcal{A}=\sum_{i=0}^{n}\left(r_{i}-1\right) E_{i}
$$

is the adjunction divisor of $\mathcal{C}$. Using Section 3.6, it can be shown that $\mathcal{A}$ is in fact rational over $k$.

- Let $\mathcal{C}^{\prime}:\{G=0\}$ be a projective plane curve defined over $k$ such that the form $G \in$ $k[X, Y, Z]$ is not divisible by $F$. Then $\mathcal{C}^{\prime}$ is an adjoint of $\mathcal{C}$ if

$$
(G) \geq \mathcal{A}
$$

If we consider the points $P_{i}$ which belong to the sub-tree $\mathcal{T}_{P}$ corresponding to a singular point $P$ of $\mathcal{C}$ and set

$$
\mathcal{A}_{P}=\sum_{P_{i} \in \mathcal{T}_{P}}\left(r_{i}-1\right) E_{i}
$$

then $\mathcal{A}_{P}$ is called the adjunction divisor of $\mathcal{C}$ at $P$. If $P$ is a simple point, then clearly $\mathcal{A}_{P}=0$. Let $G$ be a form not divisible by $F$ and such that $(G)_{P} \geq \mathcal{A}_{P}$. We say that $\mathcal{C}^{\prime}:\{G=0\}$ is an adjoint of $\mathcal{C}$ at $P$. Using the fact that $\operatorname{deg} E_{i}=r_{i}$, we can rewrite formula (6): if $\operatorname{deg} F=m$, the genus of $\dot{\mathcal{C}}$ is equal to

$$
\begin{aligned}
g & =\frac{(m-1)(m-2)}{2}-\sum_{P \in \mathcal{C}} \frac{\operatorname{deg} \mathcal{A}_{P}}{2} \\
& =\frac{(m-1)(m-2)}{2}-\frac{\operatorname{deg} \mathcal{A}}{2} .
\end{aligned}
$$

Finally let us recall the link between adjoint curves and special divisors. Let $\mathcal{C}^{\prime}:\{G=0\}$ be an adjoint curve of $\mathcal{C}:\{F=0\}, d$ (resp. $m$ ) the degree of $G$ (resp. $F$ ); we set

$$
\mathcal{R}=(G)-\mathcal{A}
$$

The divisor $\mathcal{R}$ is positive and $k$-rational. We say that $\mathcal{R}$ is the divisor cut out on $\mathcal{C}$ by the adjoint $\mathcal{C}^{\prime}$ outside $\mathcal{A}$. The following result is well known (see $[6, \mathrm{p}, 437]$ )

Theorem 4.1 The adjoint curves of degree $d=m-3$ cut out on $\mathcal{C}$ outside $\mathcal{A}$ the complete canonical series denoted by $\mathcal{K}$.

This means that any special divisor $\mathcal{R}$ of $k(\mathcal{C})$ is such that

$$
\mathcal{R}=(G)-\mathcal{A}
$$

where $G$ is a form of degree $m-3$. 


\subsection{Conductor}

In this section we refer to $[6$, p. 430$]$ or $[15$, p. 217-]. Let $\mathcal{C}$ be a projective plane curve defined and absolutely irreducible over a field $k, P$ a $k$-rational point of $\mathcal{C}, \mathcal{O}_{P}$ the local ring at $P, \overline{\mathcal{O}}_{P}$ the integral closure of $\mathcal{O}_{P}$ in $K$.

Definition 4.1 The conductor at $P$ of the curve is the set

$$
\mathrm{c}_{P}=\left\{u \in \mathcal{O}_{P} \mid u \overline{\mathcal{O}}_{P} \subset \mathcal{O}_{P}\right\}
$$

The conductor $\mathrm{c}_{P}$ is the largest ideal in $\mathcal{O}_{P}$ which is also an ideal in $\overline{\mathcal{O}}_{P}$. If $P$ is a simple 'point, then

$$
\overline{\mathcal{O}}_{P}=\mathcal{O}_{P}
$$

so $c_{P}=\mathcal{O}_{P}$. Associated to the conductor at $P$ there are two integers $n_{P}$ and $\delta_{P}$ which are important invariants of a singular point $P$.

1. The integer $n_{P}$. We set

$$
n_{P}=\operatorname{dim}_{k} \overline{\mathcal{O}}_{P} / c_{P}
$$

Since

$$
\overline{\mathcal{O}}_{P}=\bigcap_{p>P} \mathcal{O}_{p}
$$

and since $c_{P}$ is an ideal of the semi-local ring $\overline{\mathcal{O}}_{P}$, for any place $\varphi$ above $P$, the ideal generated by $c_{P}$ in the discrete valuation ring $\mathcal{O}_{\wp}$ is a power of $\wp$

$$
c_{P} \mathcal{O}_{p}=\wp^{n_{p}}
$$

and

$$
n_{P}=\sum_{p \succ P} n_{p} \operatorname{deg} \wp
$$

The conductor at $P$ is related to the divisor

$$
A_{P}=\sum_{p \succ P} n_{p} \wp
$$

in the following way

$$
\mathrm{c}_{P}=\left\{u \in \mathcal{O}_{P} \mid(u)_{P} \geq A_{P}\right\} .
$$

Observe that $\operatorname{deg} A_{P}=n_{P}$ and if $P$ is a simple point, then $n_{P}=0$ and $A_{P}=0$. In [15], $A=\sum_{P \in \mathcal{C}} A_{P}$ is called the divisor of double points of the curve. It can be shown that, since $\mathcal{C}$ is a plane curve, we have $A_{P}=\mathcal{A}_{P}$ for all $P$ in $\mathcal{C}$ so $A=\mathcal{A}$ (see [6]). If $u$ is a non zero element of $K$ then $u=\bar{G} / \bar{H}$, where $G$ and $H$ are forms of equal degree and $\bar{H} \neq 0$. We have

$$
u \in \mathrm{c}_{P} \Longleftrightarrow(G)_{P} \geq(H)_{P}+A_{P}
$$

This implies that the plane curve with equation $G=0$ is an adjoint of $\mathcal{C}$ at $P$. 
2. The integer $\delta_{P}$. The second integer associated to a point $P$ of the curve is

$$
\delta_{P}=\operatorname{dim}_{k} \overline{\mathcal{O}}_{P / \mathcal{O}_{P}}
$$

if $P$ is a simple point, then $\delta_{P}=0$ and this condition characterizes simple points. Since $\mathcal{C}$ is a plane curve, the integer $\delta_{P}$ is related to $n_{P}$ by the equality

$$
n_{P}=2 \delta_{P} \text {. }
$$

If the equation of the plane curve $\mathcal{C}$ is a form of degree $m$, then the genus of the curve is given by (compare with (7))

$$
g=\frac{(m-1)(m-2)}{2}-\sum_{P \in \mathcal{C}} \delta_{P} .
$$

Further $\delta_{P}=\sum_{Q \in \mathcal{T}_{P}} r_{Q}\left(r_{Q}-1\right) / 2$ is the number of independent conditions that must be verified by a plane curve $\mathcal{C}^{\prime}$ to be an adjoint of $\mathcal{C}$ at $P$.

\subsection{Basis for $\mathcal{L}(D)$}

The construction of a basis for $\mathcal{L}(D)$ is treated in [9] in the case where $\mathcal{C}$ has only ordinary singular points and in [12] for any singular curve. Following [12], we use the fundamental theorem of Max Noether:

Theorem 4.2 Let $G=0$ be the equation of a plane curve not containing $\mathcal{C}:\{F=0\}$ as a component. Then, if $(H) \geq \mathcal{A}+(G)$, there exist forms $A$ and $B$ with coefficients in $k$ such that

$$
H=A F+B G .
$$

Theorem 4.3 (see [12]) Let $\mathcal{C}:\{F=0\}$ be a projective plane curve defined and absolutely irreducible over $k, \mathcal{A}$ its adjunction divisor and $D$ any positive divisor in $K$. If $G_{0}$ is a form in $k[X, Y, Z]$ of degree $d$ such that $\left(G_{0}\right) \geq \mathcal{A}+D$, then

$$
\mathcal{L}(D)=\left\{\bar{G} / \bar{G}_{0} \mid G \in k[X, Y, Z], G \text { is a form, } \operatorname{deg} G=d,(G) \geq\left(G_{0}\right)-D\right\} \cup\{0\} .
$$

So, the elements of $\mathcal{L}(D)$ are related to the adjoints of the curve. To obtain a basis for this $k$-vector space, we pick a form $G_{0}$ such that $\left(G_{0}\right) \geq \mathcal{A}+D$. Suppose that $\operatorname{deg} G_{0}=d$ and

$$
\left(G_{0}\right)=\mathcal{A}+D+R
$$

then we search a basis for the $k$-vector space of the forms of degree $d$

$$
G=\sum_{i+j+l=d} \lambda_{i, j, l} X^{i} Y^{j} Z^{l}, \lambda_{i, j, l} \in k
$$

such that $G$ is not divisible by the equation of $\mathcal{C}$ and $(G) \geq \mathcal{A}+R$.

Notice that if $D$ is not positive, we can write

$$
D=D_{+}-D_{-}
$$

where $D_{+}$and $D_{-}$are positive divisors with disjoint supports. It follows that we can obtain a basis for $\mathcal{L}(D)$ in the following way: 
Theorem 4.4 If $G_{0}$ is a form in $k[X, Y, Z]$ of degree d such that $\left(G_{0}\right) \geq \mathcal{A}+D_{+}$, then

$$
\mathcal{L}(D)=\left\{\bar{G} / \bar{G}_{0} \mid G \in k[X, Y, Z], G \text { is a form }, \operatorname{deg} G=d,(G) \geq\left(G_{0}\right)-D\right\} \cup\{0\} .
$$

Proof: If $D_{-}=0$, we recover the case $D$ positive. Otherwise we set

$$
\left(G_{0}\right)=\mathcal{A}+D_{+}+R
$$

If $u=\bar{G} / \overline{G_{0}}$ where $G$ is a form of degree $d$ such that $(G) \geq\left(G_{0}\right)-D$, then

$$
(u)=(G)-\left(G_{0}\right) \geq-D
$$

and $u$ is in $\mathcal{L}(D)$. Conversely, if $u$ is a non zero element in $\mathcal{L}(D)$, then $(u)+D=D^{\prime}$ is an positive divisor and

$$
(u)+D_{+}=D^{\prime}+D_{-} ;
$$

so $D_{+}$and $D^{\prime}+D_{-}$are two positive equivalent divisors such that, if $u=\bar{H} / \overline{H^{\prime}}$, with $H$ and $H^{\prime}$ forms of equal degree, then

$$
(H)+D_{+}=\left(H^{\prime}\right)+D^{\prime}+D_{-} .
$$

Since we have

$$
\begin{aligned}
\left(G_{0} H\right) & =\left(G_{0}\right)+(H) \\
& =\mathcal{A}+D_{+}+R+(H) \\
& =\mathcal{A}+D^{\prime}+D_{-}+R+\left(H^{\prime}\right) \geq \mathcal{A}+\left(H^{\prime}\right)
\end{aligned}
$$

we may apply Max Noether's Theorem to the form $G_{0} H$ by which there exist forms $A$ and $G$ such that

$$
G_{0} H=A F+G H^{\prime}
$$

so $(u)=(H)-\left(H^{\prime}\right)=(G)-\left(G_{0}\right)$ and $u=\alpha \bar{G} / \bar{G}_{0}$, for some $\alpha \in k$.

Finally, to obtain a basis for $\mathcal{L}(D)$, we must know

1. how to compute the divisor of a given form,

2. how to find a form of given degree whose divisor is greater than a given positive divisor.

We will address these problems in the next Section.

\section{Algorithmic part}

Let $\mathcal{C}$ be a plane projective curve defined over $k=\mathbb{F}_{q}$ with equation $F(X, Y, Z)=0, \mathcal{P}$ and $D$ two $k$-rational divisors; in order to construct the code $C_{L}(\mathcal{X}, \mathcal{P}, D)$, where $\mathcal{X}$ is the smooth model of $\mathcal{C}$, we need

1. all the singular points and the $k$-rational points of $\mathcal{C}$; 
2. the desingularization tree;

3. the adjunction divisor $\mathcal{A}$, a $k$-rational basis $\left\{u_{1}, u_{2}, \ldots, u_{\ell(D)}\right\}$ for $\mathcal{L}(D)$ and the values of $u_{i}$ at the $k$-rational points $P$ occuring in the support of $\mathcal{P}$.

For this we need algorithms

(a) to compute the divisor associated to a form;

(b) to compute the order of a function $u$ at a point $P$ of the smooth model and $u(P)$ if it is defined;

(c) to interpolate forms through $k$-rational divisors: that is to find all the forms $G$ such that $(G)$ is greater than a given $k$-rational divisor.

\subsection{Finding singular and rational points}

Let $F_{X}, F_{Y}$ and $F_{Z}$ be respectively the derivatives of $F$ with respect to the variables $X, Y$ and $Z$. Then

$$
(a: b: c) \in \mathcal{C} \text { is singular } \Longleftrightarrow(\text { Sing })\left\{\begin{aligned}
F(a, b, c) & =0 \\
F_{X}(a, b, c) & =0 \\
F_{Y}(a, b, c) & =0 \\
F_{Z}(a, b, c) & =0 \\
(a, b, c) & \neq(0,0,0) .
\end{aligned}\right.
$$

To solve (Sing) it is sufficient to solve the two following systems:

$$
\left\{\begin{array}{r}
F(a, b, 1)=0 \\
F_{X}(a, b, 1)=0 \\
F_{Y}(a, b, 1)=0
\end{array}\right.
$$

and

$$
\left\{\begin{aligned}
F(a, 1,0) & =0 \\
F_{X}(a, 1,0) & =0 \\
F_{Z}(a, 1,0) & =0
\end{aligned}\right.
$$

and finally test if $(1: 0: 0)$ is a singular point. For the last step we employ the following equivalence

$$
(1: 0: 0) \in \mathcal{C} \text { is singular } \Longleftrightarrow\left\{\begin{aligned}
F(1,0,0) & =0 \\
F_{Y}(1,0,0) & =0 \\
F_{Z}(1,0,0) & =0 .
\end{aligned}\right.
$$

The algebraic systems (9) and (10) yield a finite number of solutions. Those of (10) may be found by computing the greatest common divisor

$$
R(a)=\operatorname{gcd}\left\{F(a, 1,0), F_{X}(a, 1,0), F_{Z}(a, 1,0)\right\}
$$

and the roots of $R$ yields the solutions. If $R$ does not factor linearly over $k$ then there are singular points over a finite extension of $k$. To find the solution of (9), the best method is 
to compute a Gröbner basis of the ideal generated by the polynomials occuring in (9) (see [11]).

To find the rational points we use the same idea:

$$
P=(a: b: c) \in \mathcal{C} \text { is rational over } \mathbb{F}_{q} \Longleftrightarrow(\text { Rat })\left\{\begin{aligned}
F(a, b, c) & =0 \\
a^{q}-a & =0 \\
b^{q}-b & =0 \\
c^{q}-c & =0 \\
(a, b, c) & \neq(0,0,0)
\end{aligned}\right.
$$

\subsection{Computing the desingularization tree}

Suppose that $\mathcal{C}$ has a desingularization over $k_{r}=\mathbb{F}_{q}$. Let $P=(a: b: 1)$ be a $k_{r}$-rational singular point of $\mathcal{C}$. We compute the desingularization tree by taking advantage of the action of the Galois group $\mathcal{G} a l\left(k_{r} / k\right)$, as described in Section 3.6. Instead of computing each desingularization tree for the conjugate points of $P$ if any, we compute only one tree on which the nodes will have the necessary informations concerning the conjugate points. For this purpose we fix $\sigma$ a generator of the Galois group $\mathcal{G} a l\left(k_{r} / k\right)$ and we associate to a point $P=(a, b) \in \mathbb{A}^{2}\left(k_{r}\right)$ its orbit under the action of $\sigma$ which will be represented by

$$
\mathcal{O} \mathcal{R}_{P}=\left\{0,1,2, \ldots, i_{P}-1\right\}
$$

where $i_{P}$ is the smallest positive integer such that $P^{\sigma^{i} P}=P$. The nodes of a sub-tree of $\mathcal{T}_{P}$ will be represented by

$$
\left[Q=\left(a^{\prime}, b^{\prime}\right), f_{Q}=0, r_{Q}, \mathcal{E}_{Q}:\left\{l_{Q}=0\right\}, \mathcal{O} \mathcal{R}_{Q}\right]
$$

where, in the appropriate affine neighbourhood, $Q=\left(a^{\prime}, b^{\prime}\right) \in \mathbb{A}^{2}\left(k_{r}\right)$ is an infinitely near point of multiplicity $r_{Q}$ on the strict transform with affine equation $f_{Q}=0, l_{Q}=0$ is the affine equation of the exceptional line and $\mathcal{O} \mathcal{R}_{Q}$ is the orbit of $Q$. The root $P$ of the desingularization tree $\mathcal{T}_{P}$ has the same representation but, since for $P$ the exceptional line is not defined, we write

$$
\left[P=(a, b), f_{P}=0, r_{P}, \mathcal{E}_{P}:\{\}, \mathcal{O} \mathcal{R}_{P}\right]
$$

We have the following algorithm

\section{Algorithm 5.1}

Input: $\left(P=(a, b), f_{P}, \mathcal{E}_{P}\right)$

Output: The desingularization tree of $P$. desingTree $\left(P=(a, b), f_{P}, \mathcal{E}_{P}\right)$

1. Compute $\mathcal{O R}_{P}$.

2. Set $f(x, y):=f_{P}(x+a, y+b)$; take the affine change of coordinates so that the point $P=(a, b)$ is brought to the origin. Compute $r_{P}$ which is the degree of the initial form of $f$. 
3. If $r_{P}=1$ then return the tree consisting of the single node

$$
\left[P=(a, b), f_{P}, 1, \mathcal{E}_{P}, \mathcal{O} \mathcal{R}_{P}\right]
$$

4. otherwise, let $f_{x}=0$ be the equation of the strict transform of $f$ in $U_{x}$.

5. Factor $f_{x}(0, T)=\prod_{j=1}^{t} p_{j}(T)^{m_{i}}$ and set $s=$ lowest common multiple of $\operatorname{deg} p_{j}, j=$ $1,2, \ldots t$. If $s \neq 1$ this means that there are singular points nonrational over $k$. Restart with an extension of $k$ of degree $s$.

6. Compute $R=\left\{\alpha \mid f_{x}(0, \alpha)=0\right\}$. Initialize an empty set listOfSubTrees which at the end will contain all the sub-trees of $\mathcal{T}_{P}$.

7. While $R$ is not empty repeat

(a) Pick a root $\alpha \in R$,

(b) compute $\mathcal{T}_{Q}=$ desing $\operatorname{Tree}\left(Q(0, \alpha), f_{x},\{x=0\}\right)$ and add the result to ListOfSubTrees,

(c) compute $R=R \backslash\left\{\alpha^{q^{i}} \mid i \in \mathcal{O} \mathcal{R}_{Q}\right\}$. (On the node of $\mathcal{T}_{Q}$ we may retreive $\mathcal{O} \mathcal{R}_{Q}$ )

8. Test if $x$ divides the minimal form of $f$; if so, then $Q^{\prime}=(0,0)$ is an infinitely near point in $U_{y}$; compute $\mathcal{T}_{Q^{\prime}}=$ desingTree $\left(Q^{\prime}, f_{y},\{y=0\}\right)$ where $f_{y}=0$ is the equation of the strict transform in $U_{y}$ and add the result in ListOfSubTrees.

9. Return

$$
\mathcal{T}_{P}=\left\{\left[P=(a, b), f_{P}, r, \mathcal{E}_{P}, \mathcal{O} \mathcal{R}_{P}\right], \text { listOfSubTrees }\right\}
$$

We will see in the next Section how we can take advantage of the orbit assigned to each node.

\subsection{Divisor associated to a form}

Let $G \in k[X, Y, Z]$ be a form such that $\bar{G} \neq 0$ in $\Gamma(\mathcal{C})$. For any point $P \in \mathcal{C}$, we set $g=\bar{G}^{P}$ (see Section 2) and $(G)_{P}=\sum_{p>P} \operatorname{ord}_{\mathfrak{p}}(g) \wp$. Since $(G)_{P}$ depends only on $g$, we will sometimes set $(G)_{P}=(g)_{P}$. If $G(P) \neq 0$, that is if $P$ is not a point of the curve $\mathcal{C}^{\prime}:\{G=0\}$, then $(G)_{P}=0$. The divisor of $G$ in $k(\mathcal{C})$ is equal to

$$
(G)=\sum_{P \in \mathcal{C}}(G)_{P}=\sum_{P \in \mathcal{C} \cap \mathcal{C}^{\prime}}(G)_{P}
$$

its degree is equal to $\operatorname{deg} F \times \operatorname{deg} G$ by Bézout Theorem. Let us compute $(G)_{P}$ for all the common points $P$ of $\mathcal{C}$ and $\mathcal{C}^{\prime}$.

1. If $P$ is a simple point of $\mathcal{C}$, we have

$$
(G)_{P}=\operatorname{ord}_{P}(g) P
$$


2. If $P$ is a singular point of $\mathcal{C}$, let $\pi$ be its blowing-up morphism. For each infinitely near point $Q$ of $P(\pi(Q)=P)$, let $\pi_{Q}^{*}$ be the monoidal transformation with respect to the neighbourhood $\left(U_{x}\right.$ or $\left.U_{y}\right)$ containing $Q$; let $L_{Q}=0$ be the affine equation at $Q$ of the exceptional line and $g_{Q}$ (resp. $l_{Q}$ ) the residual image of the strict transform of $G$ (resp. $\left.L_{Q}\right)$ in $\Gamma\left(\mathcal{C}_{1}\right)$. Then $(G)_{P}$ is defined in a recursive way by

$$
\begin{aligned}
(G)_{P}=(g)_{P} & =\sum_{\pi(Q)=P}\left(\pi_{Q}^{*}(g)\right)_{Q} \\
& =\sum_{\pi(Q)=P}\left(l_{Q}^{\mathrm{m}_{P}(G)} g_{Q}\right)_{Q} \\
& =\mathrm{m}_{P}(G) \sum_{\pi(Q)=P}\left(l_{Q}\right)_{Q}+\sum_{\pi(Q)=P}\left(g_{Q}\right)_{Q} \\
(g)_{P} & =\mathrm{m}_{P}(G) E_{P}+\sum_{\pi(Q)=P}\left(g_{Q}\right)_{Q}
\end{aligned}
$$

where $E_{P}=\sum_{\pi(Q)=P}\left(l_{Q}\right)_{Q}$ is the exceptional divisor of $\pi$. To each singular infinitely near point $Q$ of $P$ we associate an exceptional divisor $E_{Q}$ and if $Q$ is simple, we set $E_{Q}=0$.

If $P$ is a singular point, $(G)_{P}$ is computed recursively following the branches of $\mathcal{T}_{P}$. To illustrate how to use the orbit of a node in $\mathcal{T}_{P}$ we have the following algorithm, in which the details have been left out:

\section{Algorithm 5.2}

Input: $\left(g, \mathcal{T}_{P}\right)$ where $g$ is the affine residual image of a form $G$ and $\mathcal{T}_{P}$ is the desingularization tree of $P$.

Output: The divisor $(G)_{P}$.

localDivisor $\left(g, \mathcal{T}_{P}\right)$

1. If $P$ is a leaf then set $D=\operatorname{ord}_{P}(g) P$;

2. otherwise compute $D=\mathrm{m}_{P}(G) E_{P}+\sum_{\tau_{Q} \in S}$ localDivisor $\left(g_{Q}, \mathcal{T}_{Q}\right)$ where $S$ is the set of sub-trees of $\mathcal{T}_{P}$.

3. Return $\sum_{i \in \mathcal{O} \mathcal{R}_{P}} D^{\sigma^{i}}$.

Note that before using the algorithm localDivisor one must compute for each node the corresponding exceptional divisor. This is easily done with the following algorithm:

\section{Algorithm $\mathbf{5 . 3}$}

Input: $\mathcal{T}_{P}$ the desingularization tree of $P$.

Output: $T_{P}$ with attached to each node the corresponding exceptional divisor. desingTreeWithExceDiv $\left(\mathcal{T}_{P}\right)$

1. If $P$ is a leaf then attach to the node the divisor 0 and return $\mathcal{T}_{P}$; 
2. otherwise let $S$ be the set of sub-trees of $\mathcal{T}_{P}$ and recursively call the algorithm on the sub-trees;

$$
S^{\prime}=\left\{\text { desingTreeWithExceDiv }\left(\mathcal{T}_{Q}\right) \mid \mathcal{T}_{Q} \in S\right\} .
$$

Compute the divisor $E_{P}=\sum_{\tau^{\prime}{ }_{Q} \in S^{\prime}}$ localDivisor $\left(l_{Q}, \mathcal{T}_{Q}^{\prime}\right)$ and return the tree $\mathcal{T}_{P}$ with $E_{P}$ attached to the node.

We are now able to prove the following lemma, which is used in Section 4.1:

Lemma 5.1 Let $P$ be a point of an affine curve $C$ and let $r_{P}$ be its multiplicity. Then the exceptional divisor $E_{P}$ of the blowing-up of $P$ is of degree $r_{P}$.

Proof: Let $L=0$ be the equation of a line passing by $P$ but not tangent to $C$ at $P$; then

$$
\operatorname{deg}(L)_{P}=\mathbf{m}_{P}(C) \times \mathbf{m}_{P}(L)=r_{P} .
$$

Further, the strict transform of $L=0$ contains no infinitely near point of $P$. So $(L)_{P}=E_{P}$ and $\operatorname{deg}\left(E_{P}\right)=r_{P}$.

Applying formula (11), the computation of the divisor $(G)_{P}$ reduces to the computation of the order of some functions at simple points corresponding to the leaves of $\mathcal{T}_{P}$. Since the leaves of the desingularization tree $\mathcal{T}_{P}$ are in one-to-one correspondence with the places of $\bar{k}(\mathcal{C})$ lying above $P$, we have to calculate the order of a function at a place of $\bar{k}(\mathcal{C})$.

\subsection{Order of a function at a place}

Let $\wp$ be a place of $\bar{k}(\mathcal{C})$ and $k_{r}$ an extension of degree $r$ of $k$ such that $\wp$ is a place of degree one in $k_{r}(\mathcal{C})$. Let $u=\bar{G} / \bar{H} \in k_{r}(\mathcal{C})$. We have $\wp \succ P$ or $\wp=P$ for a unique $k_{r}$-rational point $P$ of $\mathcal{C}$. By the $\wp$-morphism (see Section 3.4)

$$
\operatorname{ord}_{\mathfrak{p}}(u)=\operatorname{ord}_{(0,0)}\left(\phi_{p}^{*}\left(\bar{G}^{P}\right)\right)-\operatorname{ord}_{(0,0)}\left(\phi_{p}^{*}\left(\bar{H}^{P}\right)\right)
$$

and both $\phi_{p}^{*}\left(\bar{G}^{P}\right)$ and $\phi_{p}^{*}\left(\bar{H}^{P}\right)$ are in $\Gamma\left(C^{\mathscr{D}}\right)$ where $C^{\mathscr{D}}$ is an affine plane curve defined over $k_{\mathrm{r}}$. The problem reduces to the following: compute $\operatorname{ord}_{P}(g)$ where $P=(0,0)$ is a simple point of an affine plane curve $C$ defined over $k_{r}$ and $g \in \Gamma(C)$. Let $(x, y)$ be the affine coordinates, $f(x, y)=0$ the equation of $C$ and $G(x, y) \in k_{\mathrm{r}}[x, y]$ be a representative of $g$. If $G(0,0) \neq 0$ then of course $\operatorname{ord}_{P}(g)=0$; otherwise suppose that $\{x=0\}$ is not the tangent to $C$ at $P$. Then the initial form of $C$ at $P$ is $f_{1}=a x-b y$, with $b \neq 0$; we set $\alpha=a / b$. Let $\pi$ be the blowing-up of $P$. On the exceptional line $\mathcal{E}:\{x=0\}$ there is a unique infinitely near point $Q=(0, \alpha) \in U_{x}$ which is $k_{r}$-rational. We have

$$
\operatorname{ord}_{P}(g)=\operatorname{ord}_{Q} \pi_{x}^{*}(g) .
$$

Let $C_{Q}$ be the strict transform of $C$ and let $f_{x}=0$ be its equation in $U_{x}$. Since $\{x=0\}$ is not the tangent to $C$ at $P, \mathcal{E}$ is not tangent to $C_{Q}$ at $Q$. Without ambiguity we denote also by $x$ the residual image of $x$ in $k_{r}\left[x, y_{1}\right] /\left\langle f_{x}\right\rangle$. Then $x$ is a local parameter at $Q$ and $\operatorname{ord}_{Q}(x)=1$. If $g_{Q}$ is the residual image in $k_{r}\left[x, y_{1}\right] /\left\langle f_{x}\right\rangle$ of the strict transform $G_{Q}$ of $G$, we have (see (5) in Section 3.2)

$$
\pi_{x}^{*}(g)=x^{\mathbf{m}_{P}(G)} g_{Q},
$$


so

$$
\operatorname{ord}_{P}(g)=\mathbf{m}_{P}(\dot{G})+\operatorname{ord}_{Q}\left(g_{Q}\right) .
$$

If $\mathrm{m}_{Q}\left(G_{Q}\right)=0$ we have ord $\mathrm{d}_{Q}\left(g_{Q}\right)=0$ and we are finished, otherwise we blow up the point $Q$. Clearly a finite number of blowing-ups gives the value of $\operatorname{ord}_{P}(g)$.

\subsection{Evaluation of a function at a rational place}

Let $\wp$ be a place of $k(\mathcal{C})$ of degree one. Like for the computing of the order we need only to consider $u(P)$, where $P=(0,0)$ is a simple point of an affine plane curve $C$ and $u=g(x, y) / h(x, y)$ where $g$ and $h$ are respectively the residual images in $\Gamma(C)$ of some $G, H \in k[x, y]$. One of the following three cases occurs:

1. if $\mathrm{m}_{P}(H)=0, u(P)=G(0,0) / H(0,0)$;

2. if $\mathbf{m}_{P}(G)=0$ and $\mathrm{m}_{P}(H)>0, u$ is not defined at $P$;

3. if $\mathrm{m}_{P}(G)>0$ and $\mathrm{m}_{P}(H)>0$, we do not know yet if $P$ is a pole of $u$ or not.

To deal with the third case, we use the same idea as before. We blow up $P$ and, considering the unique infinitely near point $Q$, we have (see (12))

$$
\pi_{Q}^{*}(u)=\frac{x^{\mathbf{m}_{P}(G)} g_{Q}}{x^{\mathbf{m}_{P}(H)} h_{Q}} .
$$

1. If $\mathbf{m}_{P}(G) \geq \mathbf{m}_{P}(H)$, then

$$
\pi_{Q}^{*}(u)=\frac{x^{\left(\mathbf{m}_{P}(G)-\mathbf{m}_{P}(H)\right)} g_{Q}}{h_{Q}} .
$$

Since $\operatorname{ord}_{P}(g)=\mathbf{m}_{P}(G)+\operatorname{ord}_{Q}\left(g_{Q}\right), \operatorname{ord}_{P}(h)=\mathbf{m}_{P}(H)+\operatorname{ord}_{Q}\left(h_{Q}\right)$ and $\mathbf{m}_{P}(G)>0$, $\mathbf{m}_{P}(H)>0$ we have

$$
\left\{\begin{array}{l}
\operatorname{ord}_{P}(g)>\operatorname{ord}_{Q}\left(x^{\left(\mathbf{m}_{P}(G)-\mathbf{m}_{P}(H)\right)} g_{Q}\right) \\
\operatorname{ord}_{P}(h)>\operatorname{ord}_{Q}\left(h_{Q}\right) .
\end{array}\right.
$$

2. If $\mathrm{m}_{P}(G)<\mathrm{m}_{P}(H)$, then

$$
\pi_{Q}^{*}(u)=\frac{g_{Q}}{x^{\left(\mathbf{m}_{P}(H)-\mathbf{m}_{P}(G)\right)} h_{Q}}
$$

and

$$
\left\{\begin{array}{l}
\operatorname{ord}_{P}(g)>\operatorname{ord}_{Q}\left(g_{Q}\right) \\
\operatorname{ord}_{P}(h)>\operatorname{ord}_{Q}\left(x^{\left(\mathbf{m}_{P}(H)-\mathbf{m}_{P}(G)\right)} h_{Q}\right) .
\end{array}\right.
$$

Thus, after a finite number of blowing-ups we recover one of the first two cases. 


\subsection{Interpolating forms through divisors}

Let $D$ be a $k$-rational effective divisor of $k(\mathcal{C})$; we want to find all the forms $G \in k[X, Y, Z]$ of a fixed degree $d$ such that $(G) \geq D$. Assume that

$$
D=\sum_{i=1}^{m} n_{i} Q_{i}
$$

where the $Q_{i}$ are places of $k(\mathcal{C})$. Set $r_{i}=\operatorname{deg} Q_{i}$ and write (see Section 2)

$$
Q_{i}=\sum_{j=1}^{r_{i}} \wp_{i, j}
$$

where $\wp_{i, j}$ are places of degree one of $k_{r_{i}}(\mathcal{C})$. If we fix $\wp_{i}=\wp_{i, 1}$ and let $\sigma_{i}$ be a generator of the Galois group $\mathcal{G} a l\left(k_{r_{i}} / k\right)$, we can also write

$$
Q_{i}=\sum_{j=0}^{r_{i}-1} \wp_{i}^{\sigma_{i}^{j}}
$$

so

$$
D=\sum_{i=1}^{m} n_{i} \sum_{j=0}^{r_{i}-1} \wp_{i}^{\sigma_{i}^{j}}
$$

Then

$$
(G) \geq D \Longleftrightarrow \operatorname{ord}_{p_{i}}\left(\bar{G}^{P_{i}}\right) \geq n_{i}, \quad i=1,2, \ldots, m
$$

where $P_{i} \in \mathcal{C}$ is the unique $k_{r_{i}}$-rational point such that either $\wp_{i} \succ P_{i}$ or $\wp_{i}=P_{i}$. Let $\wp$ be any one of those places $\wp_{i}, r$ its degree and $P$ the corresponding point $P_{i}$. We consider the affine plane curve $C^{\infty}$ (see Section 3.4) with affine coordinates $(x, y)$. We suppose that $\{x=0\}$ is not the tangent line of $C^{\infty}$ at $(0,0)$. Then $C^{\infty}$ has a parametrization in $(0,0)^{3}$

$$
\left\{\begin{array}{l}
x(t)=t \\
y(t)=\sum_{e=1}^{\infty} a_{e} t^{e}, a_{e} \in k_{r} .
\end{array}\right.
$$

Notice that if $\wp^{\prime}$ is conjugated with $\wp$ over $k$, then the parametrizations of $C^{\triangleright}$ and $C^{\phi^{\prime}}$ are conjugated over $k$.

Now consider all the monomials $H_{l} \in k[X, Y, Z]$ of degree $d(l=1,2, \ldots, s=(d+2)(d+1) / 2)$. Since $\phi_{\mathfrak{p}}^{*}\left(\bar{H}_{l}^{P}\right)$ is in $\Gamma\left(C^{\mathfrak{p}}\right)$ we easily associate to $H_{l}$ a local power series

$$
\phi_{p}^{*}\left(\bar{H}_{l}^{P}\right)(x(t), y(t))=\sum_{e=0}^{\infty} b_{l, e} t^{e}
$$

For any integer $n$ we set

$$
\Delta_{\mathfrak{p}}\left(H_{l}, n\right)=\sum_{e<n} b_{l, e} t^{e}
$$

Then clearly

$$
\operatorname{ord}_{p}\left(\bar{H}_{l}^{P}\right) \geq n \Longleftrightarrow \Delta_{p}\left(H_{l}, n\right)=0 \text {. }
$$

\footnotetext{
${ }^{3}$ It can be obtain using rational Puiseux expansions or Hamburger-Noether expansions (see [4, 2]).
} 
Let $G \in k[X, Y, Z]$ be any form of degree $d$. We set

$$
G=\sum_{l=1}^{s} \alpha_{l} H_{l}, \quad \alpha_{l} \in k
$$

then

$$
(G) \geq D \text { or } \bar{G}=0 \text { in } \Gamma(\mathcal{C})
$$

is equivalent to

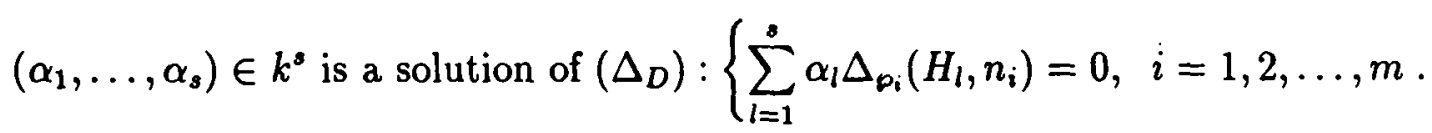

\section{Examples}

Example 6.1 Consider the plane projective curve $\mathcal{C}$ defined over $k=\mathbb{F}_{2}$ by the equation

$$
F=X^{5} Y^{2} Z^{2}+X^{2} Z^{7}+Y^{9}=0 ;
$$

$\mathcal{C}$ has two singular points, $P_{1}=(1: 0: 0)$ and $P_{2}=(0: 0: 1)$, which are in fact the only rational points of $\mathcal{C}$ over $k$. The desingularization tree $\mathcal{T}$ is composed of two sub-trees, $\mathcal{T}_{P_{1}}$ and $\mathcal{T}_{P_{2}}$

1. Sub-tree $\mathcal{T}_{P_{1}}($ here $y=Y / X$ and $z=Z / X)$

$$
\begin{aligned}
& \begin{array}{ccc}
Q_{1}=(0,0) & & Q_{2}=(0,0) \\
y_{1}^{9} z^{5}+y_{1}^{2}+z^{3} & \nu_{1}=y_{2} x & y_{2}^{9} z^{12}+y_{2}^{2}+z \\
r=2 & \mathbb{Z}=z & r=1 \\
\mathcal{E}_{P_{1}}:\{z=0\} & & \mathcal{E}_{2}:\{z=0\}
\end{array} \\
& \begin{aligned}
P_{1} & =(0,0) \\
y^{2} z^{2} & +z^{7}+y^{9} \\
r & =4
\end{aligned} \\
& y=y_{1} x \\
& \text { … } \\
& \begin{array}{ccc}
Q_{3}=(0,0) & & Q_{1}=(0,0) \\
y^{3}+y^{3} z_{1}^{7}+z_{1}^{2} & y=y & y^{8} z_{2}^{7}+y^{3}+z_{2}^{2} \\
r=2 & \overrightarrow{2}=y z_{2} & r=2 \\
\mathcal{E}_{P_{1}}:\{y=0\} & & \varepsilon_{Q_{3}}:\{y=0\}
\end{array}
\end{aligned}
$$
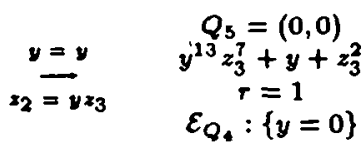

2. Sub-tree $\mathcal{T}_{P_{2}}$ (here $x=X / Z$ and $y=Y / Z$ )

$$
\begin{aligned}
& \begin{array}{ccccc}
P_{2}=(0,0) & & Q_{1}^{\prime}=(0,0) & & Q_{2}^{\prime}=(0,0) \\
x^{5} y^{2}+x^{2}+y^{9} & x=x_{1} y & x_{1}^{3} y^{5}+x_{1}^{2}+y^{7} & x_{1}=x_{2} y & x_{2}^{3} y^{8}+x_{2}^{2}+y^{5} \\
r=2 & y=y & r=2 & y=y & r=2 \\
& & \mathcal{E}_{P_{2}}:\{y=0\} & y=y & \mathcal{E}_{Q_{1}^{\prime}}:\{y=0\}
\end{array}
\end{aligned}
$$

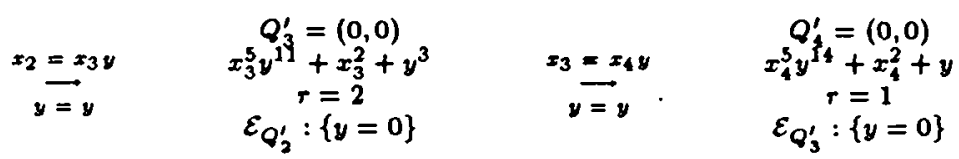


We obtain three leaves $Q_{2}, Q_{5}$ and $Q_{4}^{\prime}$ which are rational over $k$; so the smooth model of $\mathcal{C}$ has three $k$-rational points. The adjoint divisor is equal to

$$
\mathcal{A}=8 Q_{2}+10 Q_{5}+8 Q_{4}^{\prime} .
$$

Applying the genus formula (7), we have

$$
g=\frac{(9-1)(9-2)}{2}-\frac{26}{2}=15 \text {. }
$$

Using the desingularization tree we find

$$
\begin{aligned}
& \left\{\begin{array}{llll}
\operatorname{ord}_{Q_{2}}\left(\pi_{Q_{2}}^{*}(y)\right) & =\operatorname{ord}_{Q_{2}}\left(y_{2} z^{2}\right) & = & 5 \\
\operatorname{ord}_{Q_{2}}\left(\pi_{Q_{2}}^{*}(z)\right) & =\operatorname{ord}_{Q_{2}}(z) & = & 2
\end{array}\right. \\
& \left\{\begin{array}{l}
\operatorname{ord}_{Q_{5}}\left(\pi_{Q_{5}}^{*}(y)\right)=\operatorname{ord}_{Q_{5}}(y) \\
\operatorname{ord}_{Q_{5}}\left(\pi_{Q_{5}}^{*}(z)\right)=\operatorname{ord}_{Q_{3}}\left(y^{3} z_{3}\right)=7
\end{array}\right.
\end{aligned}
$$

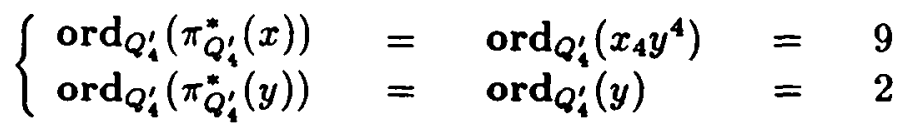

so we have

$$
\begin{aligned}
& (X)=9 Q_{4}^{\prime} \\
& (Y)=5 Q_{2}+2 Q_{5}+2 Q_{4}^{\prime} \\
& (Z)=2 Q_{2}+7 Q_{5} .
\end{aligned}
$$

Now, let $G_{0} \in k[X, Y, Z]$ be a form of degree $d \geq 7$ such that 4

$$
\left(G_{0}\right) \geq \mathcal{A} \text { and } \operatorname{supp}\left(G_{0}\right) \subseteq \operatorname{supp} \mathcal{A}
$$

and consider the $k$-rational divisor

$$
D=\left(G_{0}\right)-\mathcal{A}
$$

Then supp $D \subseteq \operatorname{supp} \mathcal{A}$ and by Bézout theorem $\operatorname{deg} D=9 d-26>2 g-2=28$; so the dimension of $\mathcal{L}(D)$ is

$$
\ell(D)=\operatorname{deg}(D)-g+1=9 d-26-15+1=9 d-40 .
$$

To obtain a basis for $\mathcal{L}(D)$, we apply Theorem 4.3 and search forms $G$ of degree $d$, not divisible by $F$ and such that

$$
(G) \geq\left(G_{0}\right)-D=\mathcal{A} \text {. }
$$

\footnotetext{
${ }^{4}$ Such a form exists: for instance we could take $G_{0}=Y^{7}$ and then $\left(G_{0}\right)=35 Q_{2}+14 Q_{5}+14 Q_{4}^{\prime}$.
} 
For this we consider the $k$-vector space $V_{d}$ which has for basis all the monomials given by the following table

\begin{tabular}{c|c}
$X^{i} Y^{j} Z^{l}, i+j+l=d$ & Number of such monomials \\
\hline$i \geq 0, j \geq 5, l \geq 0$ & $(d-4)(d-3) / 2$ \\
$i \geq 0, j=4, l \geq 1$ & $d-4$ \\
$i \geq 1, j=3, l \geq 1$ & $d-4$ \\
$i \geq 1, j=2, l \geq 1$ & $d-3$ \\
$i \geq 1, j=1, l \geq 2$ & $d-3$ \\
$i \geq 1, j=0, l \geq 4$ & $d-4$
\end{tabular}

So we have

$$
\operatorname{dim}_{k} V_{d}=(d-4)(d-2) / 2+5 d-18 .
$$

Looking at the divisors $(X),(Y)$ and $(Z)$, one can easily show that

$$
G \in V_{d} \Rightarrow \bar{G}=0 \text { or }(G) \geq \mathcal{A} \text {. }
$$

Consider the $k$-vector space

$$
V_{d}^{\prime}=\left\{G^{\prime} \in k[X, Y, Z] \mid G^{\prime} \text { a form of degree d such that } F \text { divides } G^{\prime}\right\} .
$$

Clearly, for $d=7$ or $d=8$, we have $\operatorname{dim}_{k} V_{d}^{\prime}=0$. Consider the case $d=9$. Since all the monomials occuring in $F$ belong to $V_{d}, V_{d}^{\prime}$ is a subspace of $V_{d}$. It follows easily that the same is true also for $d \geq 9$ and a basis for $V_{d}^{\prime}$ is $\{H F \mid H \in k[X, Y, Z]$ a monomial of degree $d-9\}$. Thus in any cases $(d \geq 7)$ we have

$$
\operatorname{dim}_{k} V_{d}^{\prime}=\frac{(d-7)(d-8)}{2}
$$

Hence

$$
\operatorname{dim}_{k} V_{d}-\operatorname{dim}_{k} V_{d}^{\prime}=(d-4)(d-2) / 2+5 d-18-(d-7)(d-8) / 2=9 d-40=\ell(D)
$$

so that

$$
\mathcal{L}(D)=\left\{\bar{G} / \bar{G}_{0} \mid G \in V_{d} \backslash V_{d}^{\prime}\right\} \cup\{0\}
$$

Since the divisor $D$ is rational over $\mathbb{F}_{2}$ we can use it to construct a code over any extension of $\mathbb{F}_{2}$. For instance, over $\mathbb{F}_{32}$ the curve $\mathcal{C}$ has 155 rational simple points so its smooth model has 158 rational points ${ }^{5}$. Since $\operatorname{supp} D \subseteq \operatorname{supp} \mathcal{A}$ we may take for divisor $\mathcal{P}$ the sum of the 155 rational simple points and for $d \geq 7$ and $9 d-40<155$ we may construct a class of codes with the following parameters

$$
\begin{aligned}
\text { lenght } & =155 \\
\text { dimension } & =9 d-40 \\
\text { designed distance } & =155-(9 d-26)=181-9 d .
\end{aligned}
$$

\footnotetext{
${ }^{5}$ In [10, ex. 6 p.815] it is said that the smooth model has 157 rational points over $\mathbb{F}_{32}$ and $g=26$. In the same example, for the curve with equation $X^{3} Y Z^{10}+X^{13} Z+Y^{14}=0$ one can prove that the genus is in fact $g=63$ and that there are 892 rational points on the smooth model over $\mathbb{F}_{2^{7}}$.
} 
We summarize in the following table

\begin{tabular}{c|c|c}
$d$ & dimension & designed distance \\
\hline 7 & 23 & 118 \\
8 & 32 & 109 \\
9 & 41 & 100 \\
$\vdots$ & $\vdots$ & $\vdots$ \\
20 & 140 & 1
\end{tabular}

Over $\mathbb{F}_{2^{10}}$ the curve $\mathcal{C}$ has 837 rational points, excluding the two singular points. With the same construction we obtain codes of length 837 with dimension and designed distance

\begin{tabular}{c|c|c}
$d$ & dimension & designed distance \\
\hline 7 & 23 & 800 \\
8 & 32 & 791 \\
9 & 41 & 782 \\
$\vdots$ & $\vdots$ & $\vdots$ \\
95 & 815 & 8.
\end{tabular}

Example 6.2 We construct an AG-code $C_{L}(\mathcal{X}, \mathcal{P}, D)$ where all the rational points of $\mathcal{X}$ appear in the support of $\mathcal{P}$. For that, as in $[12$, p. 248-], we consider the projective plane curve $\mathcal{C}$ defined by the equation

$$
F=X^{5}+Y^{2} Z^{3}+Y Z^{4}=0 .
$$

Over $k=\mathbb{F}_{16}$ this curve has 33 rational points with $P_{1}=(0: 1: 0)$ as a unique singular point. The desingularization tree is (with $x=X / Y$ and $z=Z / Y$ )

$$
\begin{array}{ccccc}
P_{1}=(0,0) & & Q_{1}=(0,0) & & Q_{2}=(0,0) \\
x^{5}+z^{3}+z^{4} & x=x & x^{2}+x z_{1}^{4}+z_{1}^{3} & x=x_{1} z_{1} & x_{1}^{2}+x_{1} z_{1}^{3}+z_{1} \\
r=3 & x=x z_{1} & r=2 & z_{1}=x_{1} & r=1 \\
& & \mathcal{E}_{1}:\{x=0\} & & \mathcal{E}_{2}:\left\{z_{1}=0\right\}
\end{array}
$$

and the adjunction divisor is 6

$$
\mathcal{A}=8 Q_{2} .
$$

Let $\mathcal{P}=Q_{2}+P_{2}+P_{3}+\cdots+P_{33}$ where, if $\beta$ is a primitive root of $x^{4}+x+1$, we have

$$
\begin{aligned}
& Q_{2} \succ(0: 1: 0) \quad P_{4}=(\beta: \beta: 1) \quad P_{8}=\left(\beta^{13}: \beta: 1\right) \\
& P_{2}=(0: 0: 1) \quad P_{5}=\left(\beta^{4}: \beta: 1\right) \quad P_{9}=\left(\beta^{3}: \beta^{5}: 1\right) \\
& P_{3}=(0: 1: 1) \quad P_{6}=\left(\beta^{7}: \beta: 1\right) \quad P_{10}=\left(\beta^{9}: \beta^{5}: 1\right) \\
& P_{7}=\left(\beta^{10}: \beta: 1\right) \quad P_{11}=\left(1: \beta^{10}: 1\right)
\end{aligned}
$$

and the conjugate points over $\mathbb{F}_{2}$ of $P_{4}, P_{5}, \ldots, P_{11}$. We need a $k$-rational divisor $D$ with support disjoint from the support of $\mathcal{P}$. We consider the place $\alpha$ of $k(x) / k$ associated to the irreducible polynomial $\left(x^{3}+x^{2}+1\right)$. Since $k(\mathcal{C})=k(x, y)$, where $y^{2}+y+x^{5}=0, k(\mathcal{C}) / k$

\footnotetext{
${ }^{6}$ Note that there is a misprint in [12].
} 
is an extension of degree 2 of $k(x) / k$ and there are two places $\wp_{1}, \wp_{2}$ of degree 3 in $k(\mathcal{C}) / k$ dividing $\alpha$. If $a$ is a primitive root of $x^{3}+x^{2}+1$, then we have

$$
\begin{aligned}
& \wp_{1}=\left(a: a^{2}: 1\right)+\left(a^{2}: a^{4}: 1\right)+\left(a^{4}: a: 1\right)=P_{1}^{\prime}+P_{2}^{\prime}+P_{3}^{\prime} \\
& \wp_{2}=\left(a: a^{3}: 1\right)+\left(a^{2}: a^{6}: 1\right)+\left(a^{4}: a^{5}: 1\right)=P_{4}^{\prime}+P_{5}^{\prime}+P_{6}^{\prime} .
\end{aligned}
$$

We set

$$
D=\wp_{1}+\wp_{2}
$$

which, as requested, is a $k$-rational divisor with support disjoint from supp $\mathcal{P}$. In fact, $D$ is $\mathbb{F}_{2}$-rational so that $\mathcal{L}(D)$ has a $\mathbb{F}_{2}$-rational basis. According to Theorem 4.3 we need a form $G_{0} \in \mathbb{F}_{2}[X, Y, Z]$ such that

$$
\left(G_{0}\right) \geq \mathcal{A}+D
$$

We use the method described in Section 5.6. The affine plane curve $C^{Q_{2}}$ has for equation $x_{1}^{2}+x_{1} z_{1}^{3}+z_{1}=0$ and its parametrization at $Q_{2}$ is

$$
Q_{2}:\left\{\begin{array}{l}
x_{1}(t)=t \\
z_{1}(t)=t^{2}+t^{7}+\cdots
\end{array}\right.
$$

On the affine part of $\mathcal{C}$ with $Z=1$ we find the following parametrizations at $P_{1}^{\prime}$ and $P_{4}^{\prime}$

$$
\begin{aligned}
& P_{1}^{\prime}:\left\{\begin{array}{l}
x(t)=a+t \\
y(t)=a^{2}+a^{4} t+\cdots
\end{array}\right. \\
& P_{4}^{\prime}:\left\{\begin{array}{l}
x(t)=a+t \\
y(t)=a^{3}+a^{4} t+\cdots
\end{array}\right.
\end{aligned}
$$

With the monomials of degree three, the linear system $\Delta_{A+D}$ yields a unique solution

$$
G_{0}=X^{3}+X^{2} Z+Z^{3}
$$

Indeed we have

$$
\left(G_{0}\right)=9 Q_{2}+D
$$

Next we look for the forms $G$ of degree 3 such that

$$
(G) \geq\left(G_{0}\right)-D=9 Q_{2} \text {. }
$$

Using the same method as before we have

$$
\begin{aligned}
& (X)=3 Q_{2}+P_{2}+P_{3} \\
& (Y)=5 P_{2} \\
& (Z)=5 Q_{2}
\end{aligned}
$$

and applying Theorem 4.3 it follows that a basis for $\mathcal{L}(D)$ is

$$
\mathcal{B}=\left\{\overline{X^{3}} / \bar{G}_{0}, \overline{X^{2} Z} / \bar{G}_{0}, \overline{X Z^{2}} / \bar{G}_{0}, \overline{Y Z^{2}} / \bar{G}_{0}, \overline{Z^{3}} / \bar{G}_{0}\right\} .
$$


The code $C_{L}(\mathcal{X}, \mathcal{P}, D)$ has the following parameters

$$
\begin{aligned}
\text { lenght } & =33 \\
\text { dimension } & =5 \\
\text { designed distance } & =27 .
\end{aligned}
$$

To compute the generator matrix, we evaluate the elements of $\mathcal{B}$ at the points $Q_{2}, P_{2}, \ldots, P_{11}$

\begin{tabular}{c|ccccccccccc} 
& $Q_{2}$ & $P_{2}$ & $P_{3}$ & $P_{4}$ & $P_{5}$ & $P_{6}$ & $P_{7}$ & $P_{8}$ & $P_{9}$ & $P_{10}$ & $P_{11}$ \\
\hline$\overline{X^{3}} / \bar{G}_{0}$ & 1 & 0 & 0 & $\beta^{5}$ & $\beta^{5}$ & $\beta^{4}$ & $\beta^{10}$ & $\beta$ & $\beta^{14}$ & $\beta^{7}$ & 1 \\
$\overline{X^{2}} / \bar{G}_{0}$ & 0 & 0 & 0 & $\beta^{4}$ & $\beta$ & $\beta^{12}$ & 1 & $\beta^{3}$ & $\beta^{11}$ & $\beta^{13}$ & 1 \\
$\overline{X Z^{2}} / \bar{G}_{0}$ & 0 & 0 & 0 & $\beta^{3}$ & $\beta^{12}$ & $\beta^{5}$ & $\beta^{5}$ & $\beta^{5}$ & $\beta^{8}$ & $\beta^{4}$ & 1 \\
$\overline{Y Z^{2}} / \bar{G}_{0}$ & 0 & 0 & 1 & $\beta^{3}$ & $\beta^{9}$ & $\beta^{14}$ & $\beta^{11}$ & $\beta^{8}$ & $\beta^{10}$ & 1 & $\beta^{10}$ \\
$\overline{Z^{3}} / \bar{G}_{0}$ & 0 & 1 & 1 & $\beta^{2}$ & $\beta^{8}$ & $\beta^{13}$ & $\beta^{10}$ & $\beta^{7}$ & $\beta^{5}$ & $\beta^{10}$ & 1
\end{tabular}

and add the 24 columns obtained respectively by conjugation over $\mathbb{F}_{2}$ of the columns corresponding to $P_{4}, \ldots, P_{11}$. Putting the generator matrix in its row echelon form one could easily see that the minimun distance is equal to the designed distance.

Since $\mathcal{C}$ is a hyperelliptic curve the dual code of $C_{L}(\mathcal{C}, \mathcal{P}, D)$, which is a $\left[33,5, d^{*}=4\right]$-code, can be decoded using [3].

\section{Conclusion}

Three main tasks have to be performed for the construction of an AG-code $C_{L}(\mathcal{X}, \mathcal{P}, D)$, given a plane model $\mathcal{C}$ of $\mathcal{X}$ :

1. finding all the rational and singular points of $\mathcal{C}$,

2. computing the desingularization tree of $\mathcal{C}$,

3. interpolating forms through divisors,

4. solving linear system of equations.

Those four tasks must be done over finite fields. The following activities are involved

1. Gröbner basis computation,

2. operations on polynomials such as substitution of variables and factorization,

3. operations on series which are best done using lazy evaluation: this means that only the needed terms of the series are computed,

4. linear algebra computation.

All the above are implemented in most computer algebra systems. For our implementation we have chosen AXIOM. The reason for this choice is that AXIOM is object oriented. This allows clearer and easier programming and, most of all, it enables us to define the implementation over any finite field. 


\section{References}

[1] M. Bronstein, M. Hassner, A. Vasquez and C.J. Williamson, Algebraic algorithms for the construction of error correction codes on algebraic curves, Proceedings of IEEE International Symposium on Information Theory, June 1991.

[2] A. Campillo, Algebroid Curves in Positive Characteristic, Lect. Notes in Math. 813, Springer-Verlag, 1980.

[3] I.M. Duursma, Algebraic decoding using special divisors, IEEE Trans. Info. Th., vol. 35, no 2, March 1993, 694-698.

[4] D. Duval, Rational Puiseux Expansions, Compositio Math. 70 (1989), 119-154.

[5] V.D. Goppa, Codes associated with divisors, Probl. Peredach. infor., 13(1):33-39, 1977.

[6] D. Gorenstein, An arithmetic theory of adjoint plane curves, Trans. Amer. Math. Soc. 72 (1952), 414-436.

[7] R. Hartshorne, Algebraic Geometry, Springer-Verlag 1977.

[8] H. Hironaka, On the arithmetic genera and the effective genera of algebraic curves, Memoirs of the College of Sciences of Kyoto, Series A, 30, Math. 2 (1957),177-195.

[9] M.D. Huang and D. Ierardi, Efficient algorithms for Riemann-Roch problem and for addition in the jacobian of a curve, IEEE Trans. Info. Th., July 1991, 678-687.

[10] J. Justessen, K.J. Larsen, H.E. Jensen, A. Havemose and T. Hoholdt, Construction and Decoding of a Class of Algebraic Geometry Codes, IEEE Trans. Info. Th. 35, no 4, July $1989,811-821$.

[11] D. Lazard, Solving zero-dimensional algebraic systems, J. Symbolic Cumputation, 13, 1992.

[12] D. Le Brigand and J.J. Risler, Algorithme de Brill-Noether et codes de Goppa, Bull. Soc. math. France, 116 (1988), 231-253.

[13] D. Polemi, M. Hassner, O. Moreno and C.J. Williamson, A computer algebra algorithm for the adjoint divisor, Proceedings of IEEE International Symposium on Information Theory, January 1993.

[14] H. Stichtenoth, Algebraic function fields and codes, University Text, Springer-Verlag, 1993.

[15] M:Tsfasman and S. Vladut, Algebraic-geometric codes, Kluwer Academic Pub., Math. and its Appl. 58, 1991.

[16] A.T. Vasquez, Rational desingularization of a curve defined over a finite field, Number Theory, N. Y. Seminar 1989-1990, Springer-Verlag, 229-250. 
Les rapports de recherche de l'INRIA INRIA research reports sont disponibles en format postscript sous are available in postscript format ftp.inria.fr (192.93.2.54) ftp.inria.fr (192.93.2.54)

si vous n'ave\% pas d'accès ftp if you haven't access by ftp la forme papier peut être commandée par mail : we recommend ordering them by e-mail : e-mail :dif.gesdif@inria.fr e-mail:dif.gesdif@inria.fr (n'oubliez pas de mentionner votre adresse postale). (don't forget to mention your postal address).

par courrier : by mail :

Centre de Diffusion Centre de Diffusion

INRIA INRIA

BP 105 - 78153 Le Chesnay Cedex (FRANCE) BP 105-78153 Le Chesnay Cedex (FRANCE) 
Unité de recherche INRIA Rocquencourt

Domaine de Voluceau - Rocquencourt - B.P. 105 - 78153 Le Chesnay Cedex (France)

Unité de recherche INRIA I.orraine - Technopôle de Nancy-Brahois-Campus scientifique

615, rue du Jardin Botanique - B.P. 101-54602 Villers les Nancy Cedex (France)

Unite de recherche INRIA Rennes-IRISA. Campus universitaire de Beaulieu 35042 Rennes Cedex (france)

Unite de recherche INRIA Rhone-Nipes 46, avenue Felix Viallet - 38031 Cirenoble Cedex I (France)

Unité de recherche INRIA Suphia Antipolis - 2004. route des Lucioles - B.P. 93 - 06902 Sophia Antipolis Cedex (France)

Fiditeur

INRIA - Domaine de Voluceau - Rocquencourt - B.P. 105- 78153 Le Chesnay Cedex (France)

ISSN $0249-6399$ 